\title{
Inter-domain dynamics and fibrinolytic activity of matrix metalloprotease-1
}

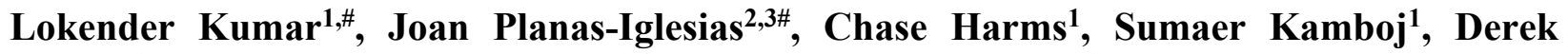 \\ Wright $^{1}$, Judith Klein-Seetharaman ${ }^{2,4, *}$, and Susanta K. Sarkar ${ }^{1, ~ *}$ \\ ${ }^{1}$ Department of Physics, Colorado School of Mines, 1500 Illinois Street, CO 80401, U.S.A. \\ ${ }^{2}$ Warwick Medical School, University of Warwick, Coventry CV4 7AL, U.K. \\ ${ }^{3}$ Loschmidt Laboratories, Department of Experimental Biology, Faculty of Science, Masaryk \\ University, Kamenice 5/A13, 62500 Brno, Czech Republic. \\ ${ }^{4}$ Department of Chemistry, Colorado School of Mines, 1500 Illinois Street, CO 80401, U.S.A.
}

${ }^{\#}$ These authors contributed equally

\author{
Corresponding author: \\ *ssarkar@mines.edu
}

\begin{abstract}
The roles of protein conformational dynamics and allostery in function are well-known. However, the roles that inter-domain dynamics have in function are not entirely understood. We used matrix metalloprotease-1 (MMP1) as a model system to study the relationship between inter-domain dynamics and activity because MMP1 has diverse substrates. Here we focus on fibrin, the primary component of a blood clot. Water-soluble fibrinogen, following cleavage by thrombin, selfpolymerize to form water-insoluble fibrin. We studied the inter-domain dynamics of MMP1 on fibrin without crosslinks using single-molecule Forster Resonance Energy Transfer (smFRET). We observed that the distance between the catalytic and hemopexin domains of MMP1 increases or decreases as the MMP1 activity increases or decreases, respectively. We modulated the activity using 1) an active site mutant (E219Q) of MMP1,2) MMP9, another member of the MMP family that increases the activity of MMP1, and 3) tetracycline, an inhibitor of MMP1. We fitted the histograms of smFRET values to a sum of two Gaussians and the autocorrelations to an exponential and power law. We modeled the dynamics as a two-state Poisson process and calculated the kinetic rates from the histograms and autocorrelations. Activity-dependent inter-domain dynamics may enable allosteric control of the MMP1 function.
\end{abstract}

Keywords: Inter-domain dynamics and activity, MMP1 conformational dynamics on fibrin, Single-molecule FRET. 


\section{Introduction}

An understanding of the relationship between the structure and dynamics of a protein is crucial for understanding its function ${ }^{1}$. Researchers have shown the correlation between the function and intra-domain dynamics within a protein domain at different hierarchies of timescales ${ }^{2-4}$. However, the roles of inter-domain dynamics in function are not entirely understood ${ }^{5,6}$. Matrix metalloproteases (MMPs) are suitable for investigating the functional relationship between interdomain dynamics and activity because the sequence of the catalytic domain remains conserved across the 23-member family ${ }^{7}$. The differences in activities among MMPs likely originate from the hemopexin domain with significant variations in the sequence ${ }^{7}$. The hemopexin domain seems to influence substrate/ligand specificity and activation/inhibition of various $\mathrm{MMPs}^{8}$. Also, prior research has reported the regulation of MMP1 catalytic activity by the hemopexin domain ${ }^{9}$. MMPs are capable of degrading numerous proteins ${ }^{10}$, including collagen, the primary component of the extracellular matrix (ECM) that provides a scaffold for cells to maintain tissue integrity ${ }^{11}$. MMPs are calcium- and zinc-dependent enzymes that are produced by cells in pro forms, i.e., they need to be activated by cleaving off the pro domain for activity ${ }^{11,12}$. MMP1, a collagenase that degrades triple-helical type-1 collagen, stands out in the 23-member MMP family because it has crystal structures available ${ }^{13}$. Also, MMP1 itself is a broad-spectrum protease, an attribute that we previously used to degrade $E$. coli proteins and purify recombinant MMP1 using a protease-based method $^{14}$. Extensive studies of MMP1 interacting with collagen monomers have revealed significant insights into the MMP1 activity, including the roles of conformational dynamics ${ }^{9,15-22}$. MMP1 consists of a catalytic domain that degrades substrates, a hemopexin domain that helps MMPs bind to the substrates, and a linker that mediates communications between the two domains (Figure 1).

Recently, we showed that inter-domain dynamics of MMP1 on type-1 collagen fibrils correlate with activity, and the two domains can allosterically communicate via the linker region as well as the substrate ${ }^{23}$. Since MMP1 is promiscuous, we investigated whether the insights into collagen fibril degradation applies to other substrates. In this paper, we focus on MMP1 inter-domain dynamics and activity on fibrin, the primary component of a blood clot. Fibrin is a critical physiological substrate because $\sim 900,000$ patients suffer from complications from clot formation leading to $\sim 300,000$ deaths $^{24}$ and $\sim \$ 28$ billion annual $\operatorname{cost}^{25}$ in the US alone. The number of adults with clot-related health issues is estimated to reach $\sim 1.82$ million by $2050^{26}$. Blood clot formation through the coagulation cascade is a part of the natural response to injury and cut and prevents loss of blood from severed blood vessels ${ }^{27}$. The life-saving process of clot

81 formation can become life-threatening if a clot

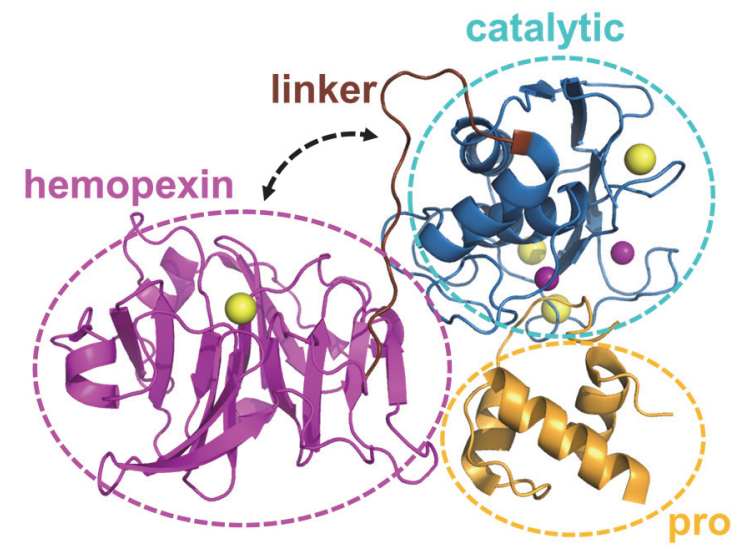

Figure 1. MMP1 structure (PDB ID: ISU3). The hemopexin domain is connected to the catalytic domain by a flexible linker. The pro domain is cleaved off to activate MMPs for catalysis. Yellow and purple spheres represent the van der Waals radii of the calcium and zinc atoms, respectively. The pro domain, the catalytic domain, the linker, and the hemopexin domains are roughly defined by the ranges of residues D32Q99, F100-Y260, G261-C278, and D279-C466, respectively. 
clot formation include heart attack, stroke, and pulmonary embolism, accounting for $\sim 50 \%$ of all hospital deaths.

Reperfusion, the act of restoring blood flow, with tissue plasminogen activator (tPA) remains the standard treatment for ischemic stroke ${ }^{28}$. Plasminogen becomes plasmin after cleavage by tPA and is able to degrade blood clots ${ }^{29}$. However, initiating tPA treatment after 3-4.5 h of stroke onset can lead to adverse side effects, notably, hemorrhagic transformation ${ }^{30}$. Therefore, it is important to explore alternative approaches that could either replace tPA treatment or extend the time window for tPA perfusion beyond the current time frame ${ }^{28}$. For example, staphopain A and staphopain B, cysteine proteases released by pathogenic Gram-positive bacteria Staphylococcus aureus, impair clotting by degrading fibrinogen ${ }^{31}$. Also, the observation of clot degradation in plasminogendeleted animals suggests alternative fibrinolytic pathways ${ }^{32}$. Many proteases other than plasmin have shown fibrinolytic activity. MMPs are potential alternatives in the fibrinolytic system because MMPs are in the blood both in pro $^{33,34}$ and activated ${ }^{35-38}$ forms. There are reports of fibrinolytic activity of MMP3 ${ }^{39}, \mathrm{MMP}^{40}$, and MMP14 ${ }^{41}$, but the field is relatively unexplored ${ }^{42}$. Prior research has reported that MMP1 does not have significant fibrinolytic activity ${ }^{39}$, which is in contrast to our observations presented in this paper.

Here we show that MMP1 inter-domain dynamics on fibrin are activity-dependent and modulated by an enhancer or an inhibitor of MMP activity. We calculated the distance between S142 in the catalytic domain and S366 in the hemopexin domain of MMP1 using smFRET measurements. The area-normalized histograms of smFRET values represent the distributions of conformations, whereas the normalized autocorrelations represent correlations between structures at different time points. From the histograms, we defined two MMP1 structures with 1) inter-domain distance of $\sim 4.5 \mathrm{~nm}$ and 2) inter-domain distance of $\sim 5.4 \mathrm{~nm}$ as the closed and open states, respectively. A comparison of active and active site mutant suggests that the open conformation of MMP1 is functionally essential. The open conformations with well-separated domains appear more in the presence of an activity enhancer (MMP9) and less in the presence of an inhibitor (tetracycline). From autocorrelations, we learned that inter-domain dynamics are not entirely random and have exponentially-distributed correlations. We fitted a sum of two Gaussians to the histograms and an exponential to the autocorrelations. We modeled the dynamics as a two-state process, where the locations of two states are the centers of Gaussian fits, and the sum of two kinetic rates between the two states is the decay rate of exponential fits. Anisotropic Network Modeling (ANM) of MMP1 dynamics revealed that a larger separation between the two domains (open conformation) often accompanies a larger catalytic pocket opening between N171 and T230. A larger catalytic pocket opening, in turn, enables closer proximity to the three chains of fibrin. ANM simulations further revealed that the $\alpha$-chain of fibrin is closest to the MMP1 active site, whereas the $\gamma$-chain is furthest. SDS PAGE of ensemble fibrin degradation confirmed that MMP1 cleaves the $\alpha$-chain first, followed by the $\beta$ - and $\gamma$ - chains. The synergistic combination of smFRET, stochastic simulations, ANM simulations, and ensemble assays provides an integrative approach to investigate the functional roles of inter-domain dynamics. This approach applies to other biochemical processes where water-soluble enzymes interact with water-insoluble substrates. 


\section{Results and discussion}

124 Single-molecule measurement of MMP1 inter-domain dynamics on fibrin without crosslinks.

125 Water-soluble fibrinogen, upon limited cleavage by thrombin, self-polymerize into water-insoluble fibrin without crosslinks. The addition of factor XIII and $\mathrm{CaCl}_{2}$ creates fibrin with crosslinks. For single molecule measurements, we used fibrin without crosslinks because we used a crystal structure of fibrin without crosslinks for simulations. Besides, fibrin without crosslinks is optically clearer than fibrin with crosslinks, which facilitates imaging with less background. To measure MMP1 inter-domain dynamics at the single molecule level, we measured smFRET between two dyes attached to the catalytic and hemopexin domains of MMP1. We mutated S142 in the catalytic domain and S366 in the hemopexin domain of MMP1 to cysteines for labeling. The distances between S142 and S366 are $\sim 5.4 \mathrm{~nm}$ and $\sim 4.5 \mathrm{~nm}$ for the open and closed conformations of MMP1, respectively. We selected a pair of dyes, Alexa555 and Alexa647, because the Forster radius between the two fluorophores is $5.1 \mathrm{~nm}$. The FRET efficiency is $50 \%$ when the distance between the fluorophores is equal to the Forster radius. More importantly, FRET is most sensitive to any distance change around the Forster radius.

A

A

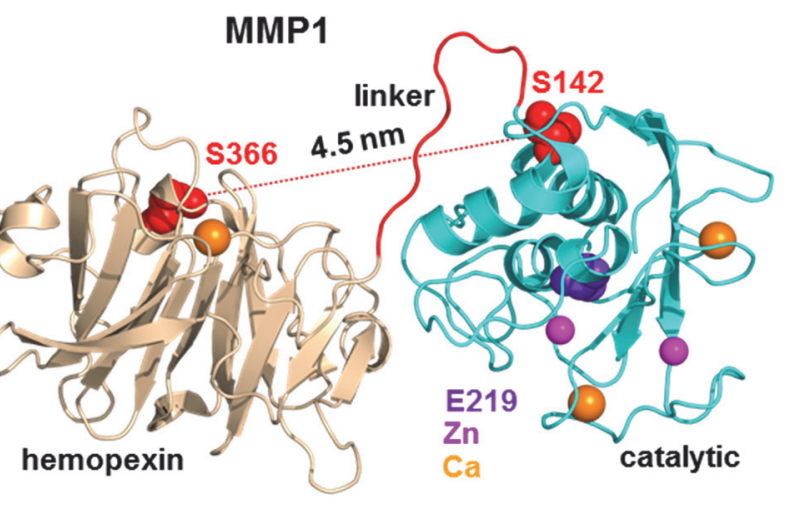

C

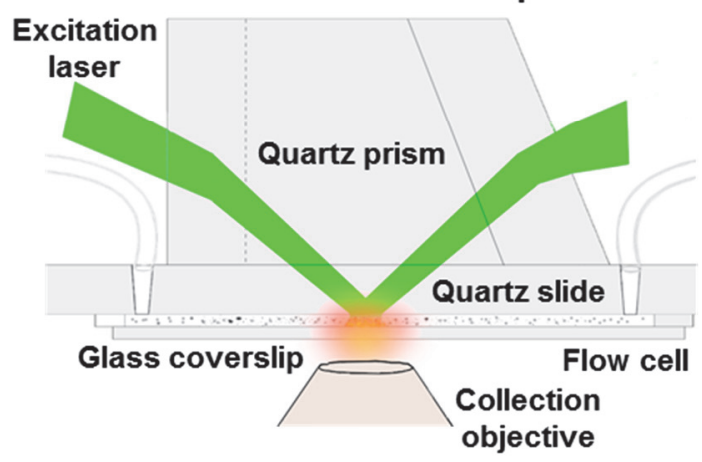

B Fibrin without crosslinks

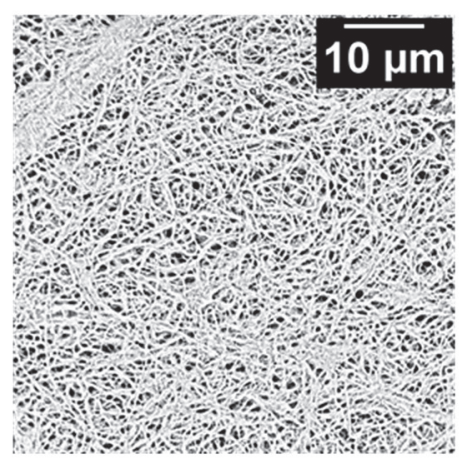

FRET trajectory
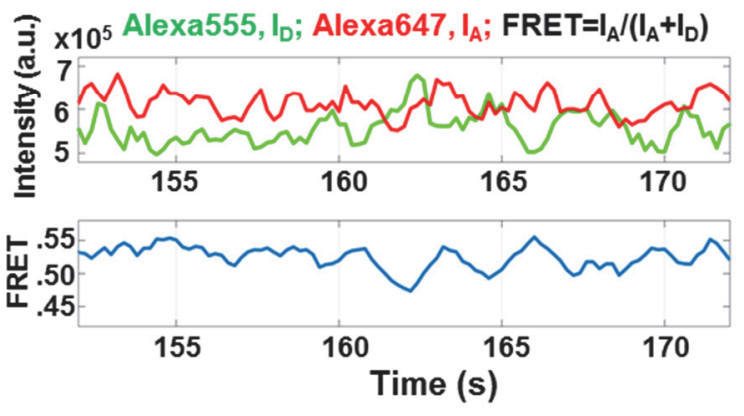

Figure 2. Single-molecule measurement of MMP1 dynamics on fibrin without crosslinks. (A) Crystal structure of MMP1 (PDB ID 1SU3). Mutations of S142 and S366 to cysteines enables attaching Alexa555 and Alexa647 dyes. (B) Scanning Electron Microscope (SEM) images of fibrin without crosslinks. (C) Schematics of the TIRF microscope used for measuring MMP1 inter-domain dynamics on fibrin. (D) Emission intensities of the two dyes. (top panel) Low FRET conformations lead to high Alexa555 emission, whereas High FRET conformations lead to Low Alexa555 emission. Anticorrelated Alexa647 and Alexa555 emissions, $\mathrm{I}_{\mathrm{A}}$ and $\mathrm{I}_{\mathrm{D}}$, respectively; (bottom panel) Calculated smFRET trajectory to show MMP1 inter-domain dynamics as a function of time. 
Single molecule measurements suffer ${ }^{43}$ from labeling ${ }^{44}$, solution conditions ${ }^{45}$, and properties of fluorophores ${ }^{46-49}$. The stochastic nature of labeling CYS142 and CYS366 can also be problem, which we discussed in our previous publication ${ }^{23}$. Since active MMP1 and active site mutant of MMP1 would be affected equally by these complications, we can distinguish the effects due to activity. Note that the amino acid E2 $19^{14}$ is the same as E200 ${ }^{13}$, which differs because we included 19 residues of the pro domain. In a previous publication, we showed that the specific activities of labeled and unlabeled MMP1 were not affected by labeling ${ }^{50}$. A flexible linker connects the catalytic and hemopexin domains of MMP1 (Figure 2A). Figure 2B shows fibrin without crosslinks. We measured MMP1 inter-domain dynamics on a thin layer of water-insoluble fibrin in a flow cell using a Total Internal Reflection Fluorescence (TIRF) microscope (Figure 2C). Figure 2D shows one smFRET trajectory. For each condition, we collected more than 300,000 smFRET values for analysis of MMP1 inter-domain dynamics on fibrin (Figure 3).
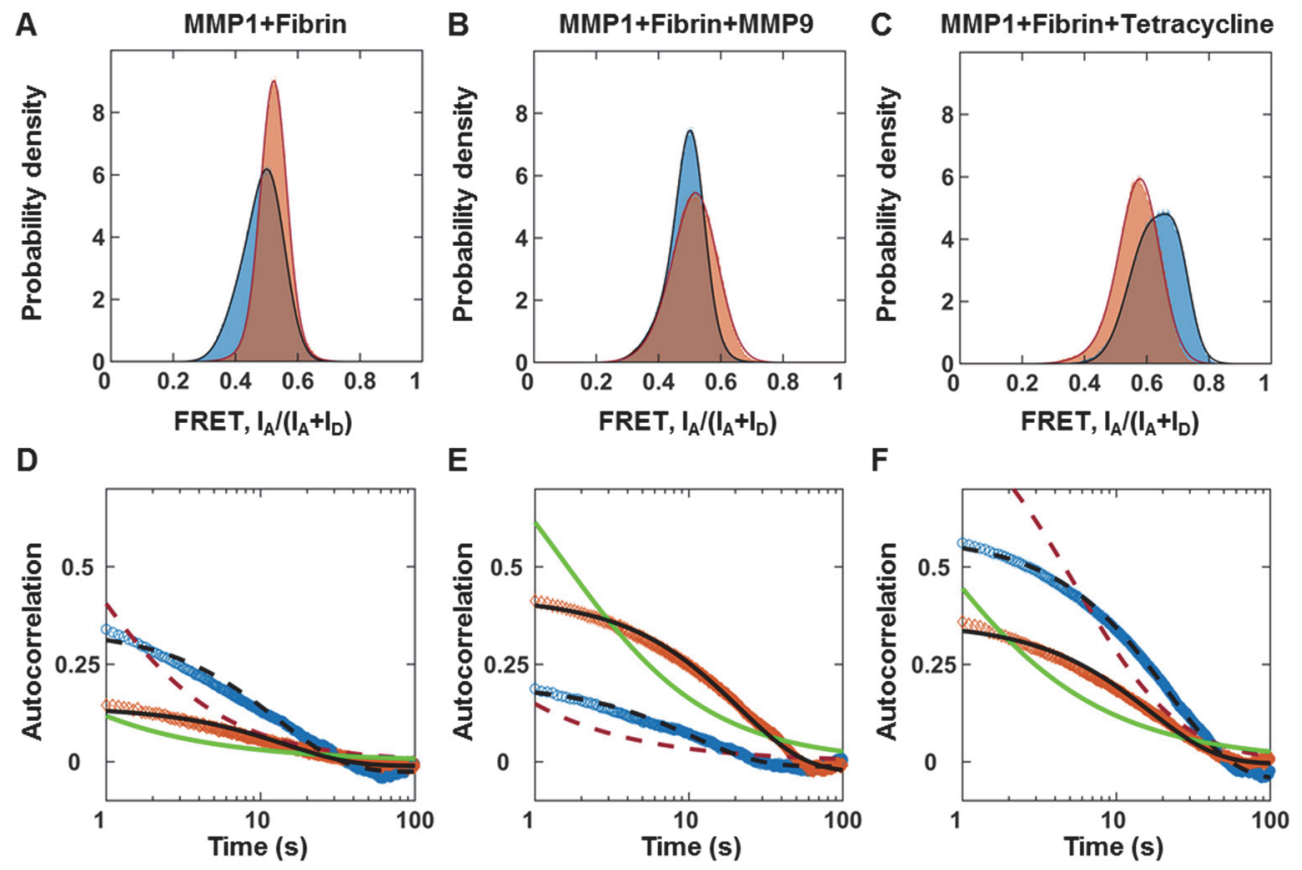

Figure 3. Inter-domain dynamics of MMP1 on fibrin correlates with activity. More than 300,000 smFRET values at $22^{\circ} \mathrm{C}$ with a $100 \mathrm{~ms}$ time resolution for each condition are used to create areanormalized histograms of MMP1 inter-domain distance (bin size=0.005). (A) Histograms without ligand, (B) Histograms in the presence of MMP9 (an enhancer), and (C) Histograms in the presence of tetracycline (an inhibitor) for active (blue) and active site mutant (orange) MMP1. Histograms are fitted to a sum of two Gaussians (active: solid blue line; active site mutant: solid red line). Autocorrelations of MMP1 inter-domain distance are calculated from the time series of smFRET values. (D) Autocorrelations without ligand, (E) Autocorrelations in the presence of MMP9, and (F) Autocorrelations in the presence of tetracycline for active MMP1 (blue) and active site mutant of MMP1 (orange). Autocorrelations are fitted to exponentials and power laws (exponential fit to active: dashed black line; power law fit to active: dashed red line; exponential fit to active site mutant: solid black line; power law fit to active site mutant: solid green line). The error bars in the histograms and autocorrelations represent the square roots of the bin counts and the standard errors of the mean (sem) and are too small to be seen. The supplementary information contains the fit equations and the best-fit parameters for histograms and autocorrelations (Table S1). We approximated smFRET efficiency by $I_{A} /\left(I_{A}+I_{D}\right)^{51}$ and has no unit. 
MMP1 inter-domain dynamics on fibrin correlate with activity. Each value of smFRET represents a conformation of MMP1. The histograms of smFRET values measuring inter-domain distances (Figure 3A) for active MMP1 and active site mutant of MMP1 suggest that low FRET conformations, where the two domains are well-separated, occur more for active MMP1 (blue histogram) compared to active site mutant (orange histogram). The prevalence of low FRET conformations for active MMP1 suggests that the two domains need to move away from each other for performing catalytic activity on fibrin. Previously, we observed the same effects for MMP1 function on type-1 collagen ${ }^{23}$. Collagen degradation by MMP1 is enhanced by MMP9 and inhibited by tetracycline. For collagen, we found that low FRET conformations appear more for MMP9 and less for tetracycline consistent with the enhancement and inhibition of MMP1 activity. To test whether or not MMP1 follows similar inter-domain dynamics when bound to fibrin, we performed smFRET measurements in the presence of MMP9 and tetracycline. In the presence of tetracycline, low FRET conformations of MMP1 on fibrin significantly disappear on fibrin (Figure 3C), as observed on type-1 collagen. Surprisingly, in the presence of MMP9, the MMP1 interdomain dynamics on fibrin (Figure 3B) show more high FRET states in contrast to the occurrence
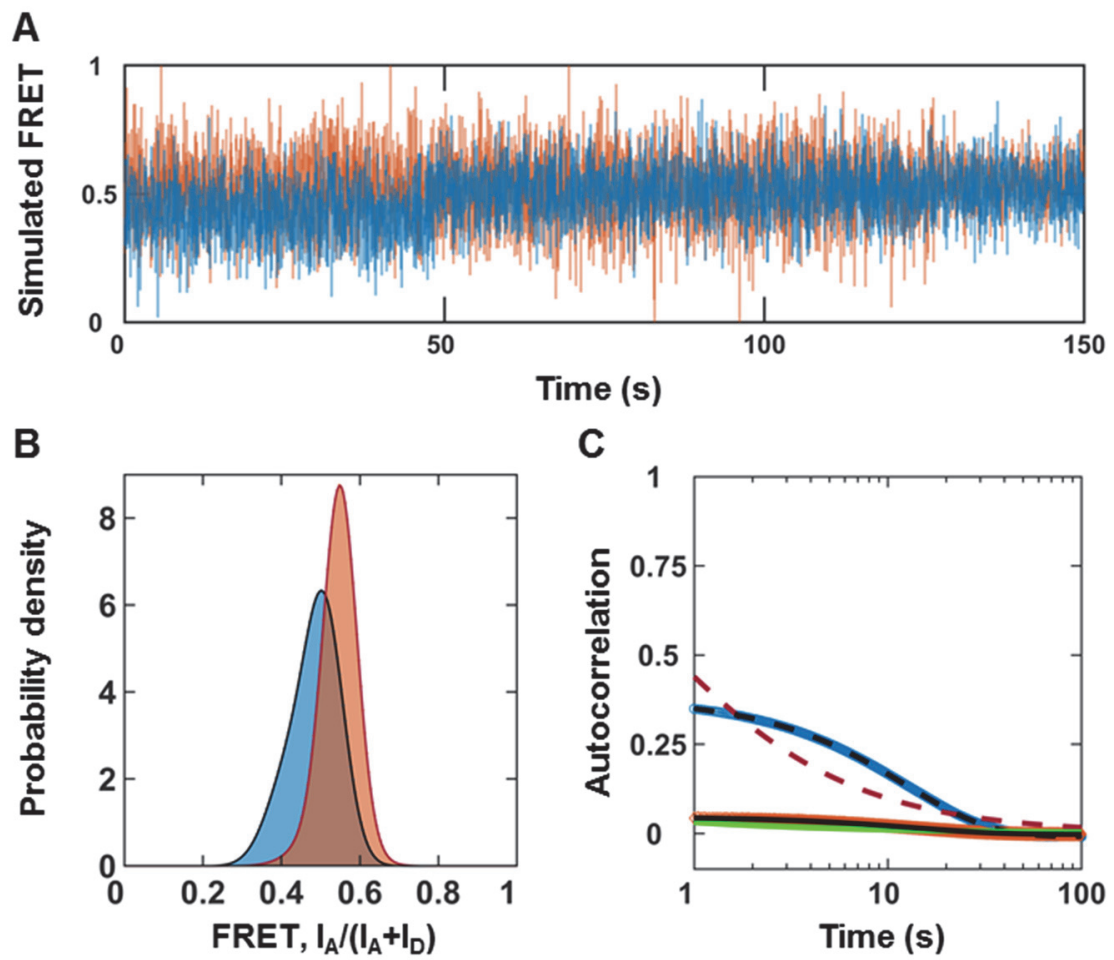

FIGURE 4. MMP1 inter-domain dynamics as a two-state system. (A) Examples of simulated smFRET trajectories with noise for active MMP1 (blue) and active site mutant of MMP1 (orange) using experimentally-determined parameters for MMP1 without tetracycline. (B) Area-normalized histograms of simulated smFRET values with best fits to a sum of two Gaussians (solid black line). (C) Autocorrelations of simulated smFRET trajectories with best fits to exponentials (active: dashed black line; active site mutant: solid black line). As expected, power law did not fit autocorrelations (active: dashed red line; active site mutant: solid green line). $\mathrm{k} 1+\mathrm{k} 2$ was recovered from exponential fits with and without noise. The error bars are the standard errors of mean for histograms and autocorrelations and are too small to be seen.

165 of more low FRET states on collagen. To determine how a conformation at one time point 166 correlates with a conformation at another time point, we calculated the autocorrelations of 
conformations (Figure 3D-F). Without any ligand, the correlation of dynamics on fibrin (Figure 3D) is higher for active MMP1 at shorter times, similar to previously reported dynamics on collagen. However, the correlations in the presence of MMP9 and tetracycline show opposite orders on fibrin (Figure 3E and 3F) and collagen (previously reported) ${ }^{23}$. The correlated motions indicate a decrease in conformational entropy. They can affect kinetics and thermodynamics of biological processes, including catalysis ${ }^{52}$. The observed modulations of correlations under different conditions suggest allosterically controlled inter-domain communications. The quantitative comparisons of best-fit parameters (Table S1) strongly indicate that inter-domain dynamics and activity of MMP1 are functionally related and allosteric. Further, a comparison with

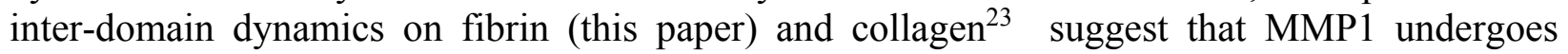
substrate-dependent and allosterically-controlled dynamics.

A two-state Poisson process describes MMP1 inter-domain dynamics on fibrin. While the histograms reveal distributions of conformations, the autocorrelations reveal whether or not conformations at different time points are related. We found that a sum of two Gaussians fits the histograms of smFRET values. Power law fits the autocorrelations for two-state simulations without noise at millisecond timescales and molecular dynamics simulations at picosecond timescales for collagen ${ }^{23}$. As such, we tried to fit both power law and exponential to the autocorrelations for fibrin. The fit equations and best-fit parameters are in the supplementary information. The exponential fits to autocorrelations enable a more straightforward interpretation of the decay rates of correlations if we approximate the conformations of MMP1 as a two-state Poisson process ${ }^{23}$. As a result, we can establish a quantitative connection between the histograms and autocorrelations to calculate the kinetic rates between the two states. To test this, we considered the two centers of Gaussian fits to histograms for MMP1 without ligands (Figure 3A) as the two states, S1 (low FRET) and S2 (high FRET). We defined the two kinetic rates as k1 $(\mathrm{S} 1 \rightarrow \mathrm{S} 2)$ and $\mathrm{k} 2(\mathrm{~S} 2 \rightarrow \mathrm{S} 1)$ for interconversion between $\mathrm{S} 1$ and $\mathrm{S} 2$. For a two-state system, we calculated k1/k2 from the ratio of Gaussian area(S2)/area(S1) and k1+k2 from the decay rates of autocorrelations. We solved the two equations to calculate k1 and k2 (Table S1C) for both active MMP1 and active site mutant of MMP1. With experimentally-determined S1, S2, k1, k2, and noise (widths of the histograms), we simulated smFRET trajectories for active MMP1 and active site mutant of MMP1 and analyzed the same way as we did experimental smFRET trajectories (Figure 4). For comparison, experimentally-determined inputs and recovered parameters from two-state simulations are in Table S2. In summary, we show that a two-state system describes MMP1 interdomain dynamics.

200 For active MMP1 without ligands, the two states are $\mathrm{S} 1=0.42$ and $\mathrm{S} 2=0.51$ on fibrin (Table S1A), which are similar to the two states $\mathrm{S} 1=0.44$ and $\mathrm{S} 2=0.55$ on collagen. However, the correlation decay rate of $0.08 \mathrm{~s}^{-1}$ on fibrin (Table S1C) is lower than the rate of $0.13 \mathrm{~s}^{-1}$ on collagen. In the presence of MMP9 and tetracycline, the two states and kinetic rates change even though a twostate description still applies for active MMP1 and active site mutant of MMP1 on fibrin (Figure 3 and Table S1) and collagen. In other words, MMP1 undergoes substrate-dependent inter-domain dynamics that follow a two-state Poisson process.

The two-state description also reveals the importance of noise in autocorrelations. Without noise, both power law and exponential fit the autocorrelations of simulated smFRET trajectories (Figure S1). With noise, however, only exponential fits the autocorrelations. Just as noise can convert a Lorentzian line shape into Gaussian line shape ${ }^{53}$, noise seems to turn a power law correlation into 
an exponential one. Note that the exponential fits recover the underlying sum of the simulated kinetic rates with and without noise.
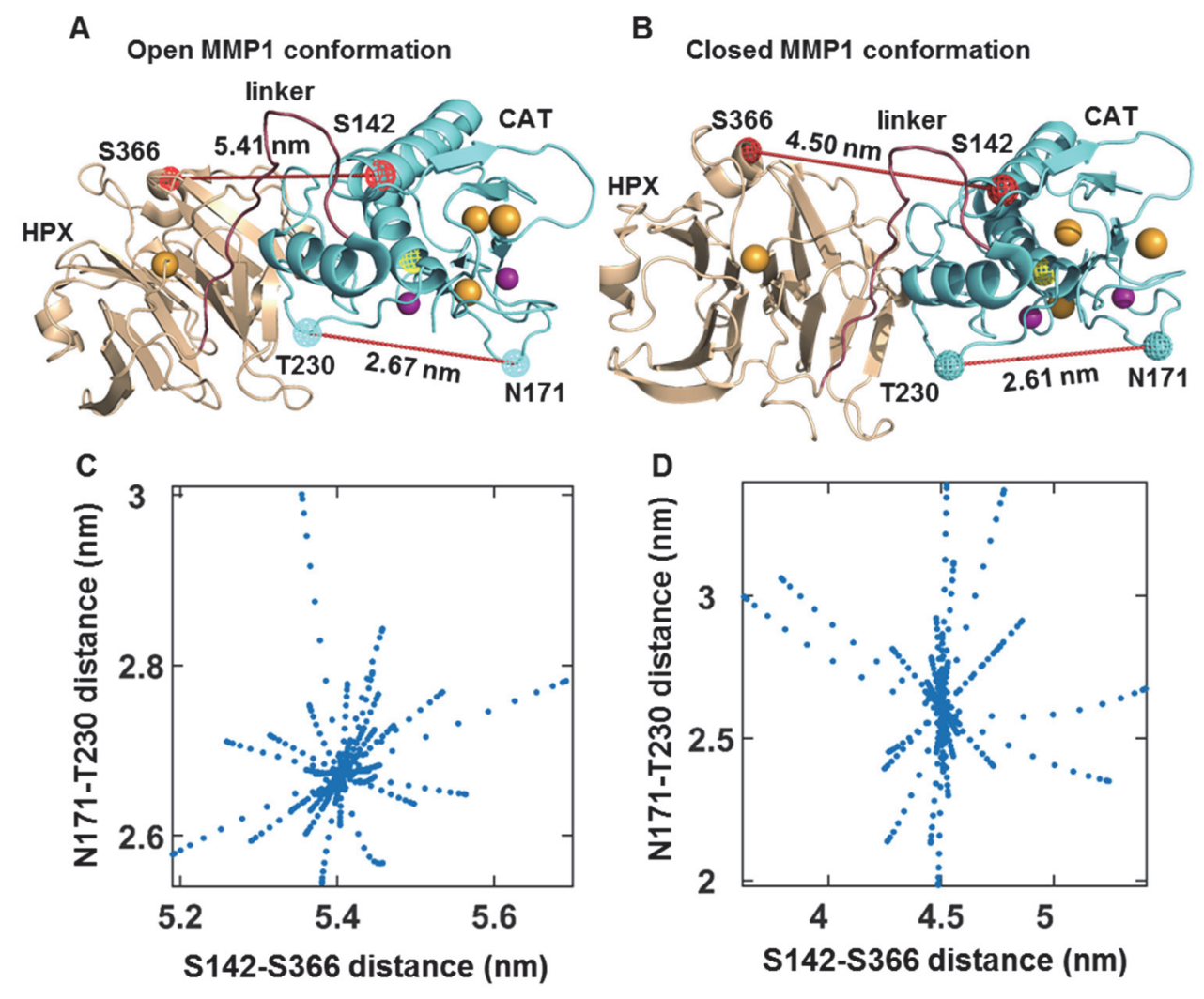

Figure 5. Correlations of MMP1 inter-domain distance with catalytic pocket opening when MMP1 is not bound to a substrate. Examples of (A) open and (B) closed conformations of MMP1. The interdomain distances between S142 and S366 and corresponding catalytic pocketing openings between N171 and T230 have been noted. Spheres and cages represent the van der Waals radii. Yellow cage: E219; Wheat sphere: calcium; Mauve sphere: zinc. (C) and (D) are scatter plots of inter-domain distance and catalytic pocket opening for the open and closed conformations, respectively. Two distances are calculated using Anisotropic Network Modeling (ANM) simulations (see methods).

213 Larger inter-domain distance can accompany larger catalytic pocket opening. The catalytic 214 cleft of MMP1 $(\sim 0.5 \mathrm{~nm})$ is narrow ${ }^{17}$, and as such, cleavage of substrates such as fibrin monomer 215 (diameter $\sim 2-5 \mathrm{~nm}$ ) ${ }^{54}$ and collagen monomer (diameter $\left.\sim 1.5 \mathrm{~nm}\right)^{55}$ needs more opening of the catalytic pocket for easier access to the catalytic cleft. To test this, we used ANM simulations ${ }^{56}$ to calculate normal modes of MMP1 dynamics. ANM models proteins as a system of beads (amino acids) connected by springs (bonds) and calculates the preferred/normal modes of protein motion and corresponding eigenfrequencies of movement. Single molecule experiments (Figure 3) suggest that both active MMP1 and active site mutant of MMP1 has an open conformation (lower FRET) and a closed conformation (higher FRET). Therefore, we chose two MMP1 conformations (Figure 5A and 5B) for ANM simulations (see Methods for details). To select the open conformation, we performed coarse-grained simulations of free MMP1 using ANM and decided on the MMP1 conformation with the highest inter-domain distance (Figure 5A). For closed conformation, we selected the conformation in the crystal structure (PDB ID: 1SU3) (Figure 5B). We considered 20 modes (20 frames for each mode) with the lowest frequencies (slower motion) 
to calculate the inter-domain distance (S142-S366) and catalytic pocket opening (N171-T230). A comparison of Figure 5C and 5D shows that MMP1 in the open conformation has an overall catalytic pocket opening $(\sim 2.7 \mathrm{~nm})$, whereas the closed structure has an opening $(\sim 2.6 \mathrm{~nm})$. Since a larger catalytic pocket opening enables access to the catalytic cleft, we can infer that larger interdomain distances (lower FRET) correlate with MMP1 activity.

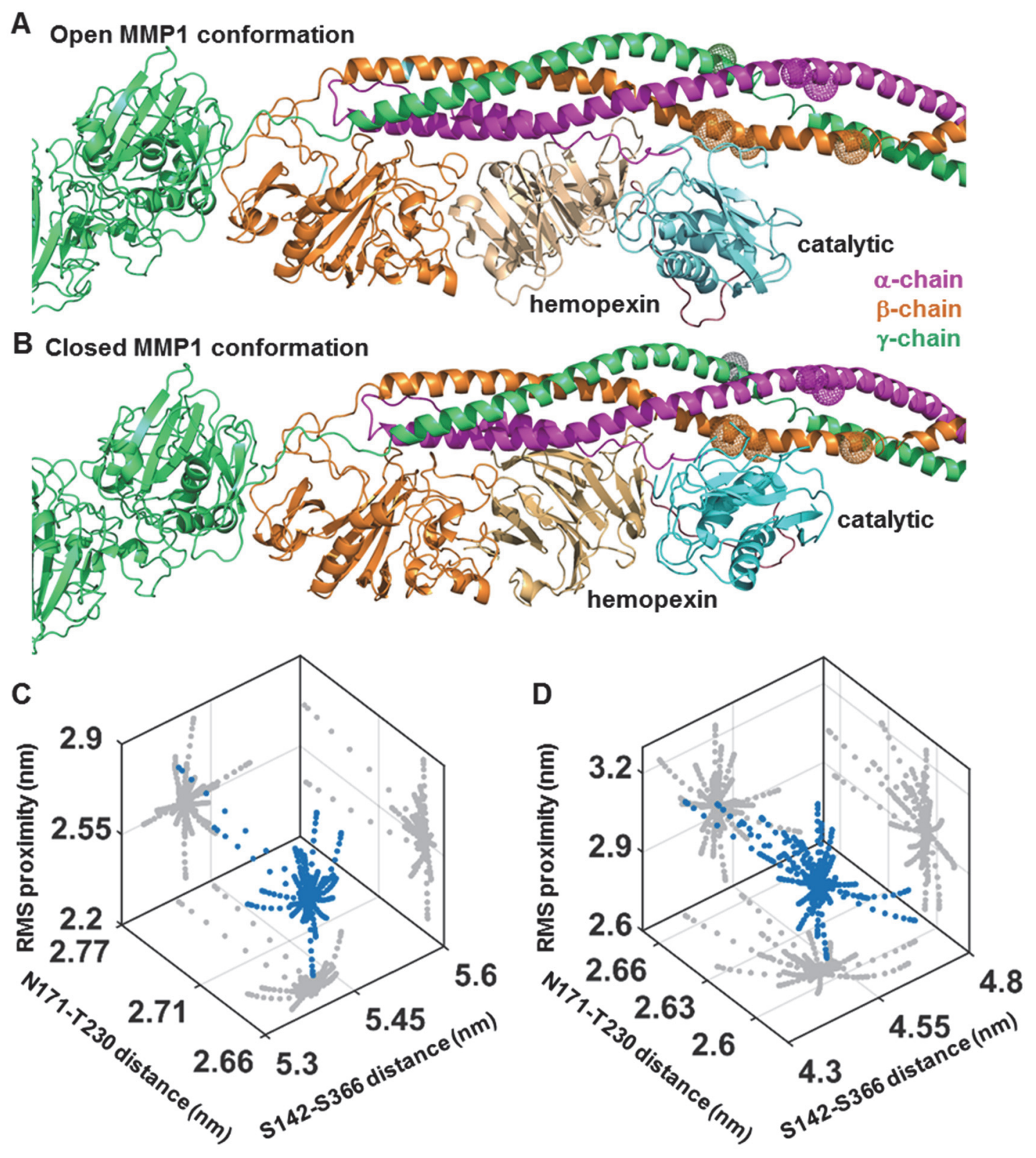

Figure 6. Correlations of MMP1 inter-domain distance with catalytic pocket opening when MMP1 is bound to the reconstructed fibrin model combining 3GHG and 1FZC. Examples of (A) open and (B) closed conformations of MMP1 bound to the reconstructed model of fibrin. Three dimensional scatter plots (blue circle) of inter-domain distance (S142-S366), catalytic pocket opening (N171-T230), and rms proximity between the MMP1 catalytic site and the three fibrin chains for (C) open and (D) closed MMP1 conformations. Two-dimensional projections of the scatter plots are in gray. The open structure shows larger catalytic pocket openings. 
To investigate whether or not a larger inter-domain distance correlates with a larger catalytic pocket opening when MMP1 is bound to fibrin, we needed to determine the docking poses of MMP1 with fibrin because there is no crystal structure of fibrin-bound MMP1. To this end, open and closed conformations of MMP1 (Figure 5A and 5B) were docked to a reconstructed model of fibrin using ClusPro ${ }^{57}$, a protein-protein docking server. The reconstructed model (see methods for detailed procedure) is a combination of the crystal structures of fibrinogen (PDB ID: 3GHG) and fibrin (PDB ID: 1FZC). The docking poses were sorted based on the distance from the $\alpha$-carbon of E219 at the MMP1 catalytic site to the potential cleaving sites on fibrin. Since the cleavage sites on fibrin for MMP1 are unknown, we considered the cleavage sites for MMP3, MMP7, and MMP14 as substitutes: the $\alpha$-chain at Asp97-Phe98, and Asn102-Asn103; the $\beta$-chain at Asp123Leu124, Ans137-Val138, and Glu141-Tyr142; and the $\gamma$-chain at Thr83-Leu84 ${ }^{58}$ (Figure S2). For each docking pose, we calculated the minimum distance possible for the three chains and considered the average of three lengths as the metric for the catalytic domain-fibrin proximity. In addition, we assumed that the hemopexin domain remains bound to the substrate, and thus, we considered the distance from the center of mass (CoM) of the hemopexin domain to any atom of the substrate as the metric for the hemopexin domain-fibrin proximity. We sorted the docking poses according to the two measures of proximity to the MMP1 domains. We selected the docking pose with minimum scores for the two criteria for ANM simulations (Figure 6A and 6B). We considered 20 modes (20 frames for each mode) with the lowest frequencies (slower motion) to calculate inter-domain distance (S142-S366), catalytic pocket opening (N171-T230), and rms proximity between the MMP1 catalytic site and the three fibrin chains (Figure 6C and 6D). The inter-domain distance reduces after binding fibrin for both the open and closed conformations of MMP1. As is the case of free MMP1 (Figure 5C and 5D), fibrin-bound MMP1 also showed that larger inter-domain distance for the open conformation accompany larger catalytic pocket openings (Figure 6C and 6D). In contrast, fibrinogen-bound MMP1 shows larger catalytic pocket opening for the closed conformation (Figure S3). The importance of open conformations on fibrin and closed conformations on fibrinogen is an experimentally testable insight from ANM simulations. At the single molecule level, we could test with fibrinogen attached to a quartz slide for TIRF imaging. Note that the overall charge is negative for both fibrinogen and a clean quartz slide, making it challenging to attach fibrinogen on a quartz slide. Nevertheless, histograms of smFRET values would be peaked at a higher FRET value on fibrinogen as compared to fibrin. One could also try to crystalize MMP1 with fibrinogen and fibrin, and check the conformations. Nevertheless, both fibrinogen- and fibrin-bound MMP1 show closer proximity to the three chains for the open conformation. The proximity suggests that a larger inter-domain distance is relevant 266 for MMP1 activity.

267 The proximity of MMP1 to the three chains correlates with the sequence of degradation. 268 Since MMP1 needs to come in the proximity before cleavage, we performed molecular docking of MMP1 with fibrin. Figure 7A shows MMP1 molecular docking poses superimposed on the reconstructed model of fibrin. MMP1 binds to fibrin at specific places in both open and closed conformations. To gain further insights, we calculated the distance between every atom in MMP1 and the three fibrin chains. We counted the number of distances below $0.5 \mathrm{~nm}$ for the top 30 docking poses and calculated the mean and standard deviation (Figure 7B). On average, MMP1 in open conformation showed the closest proximity to the $\alpha$-chain (Figure 7B, left panel). We quantified the statistical significance by p-value (Figure 7B, left panel). In contrast, the closed conformation of MMP1 did not show any significant difference in proximity to the three chains (Figure 7B, right panel). 
To test whether or not the sequence of MMP1 proximity to the three chains has any implication in fibrinolytic activity, we performed the time-dependent degradation of fibrinogen. Figure S4B shows that the overall catalytic activity is fastest on the $\alpha$-chain and slowest on the $\gamma$-chain. Fibrinogen, the basic building block of fibrin, has three pairs of amino acid chains: 1 ) the $\alpha$-chain at $\sim 63 \mathrm{kDa}, 2)$ the $\beta$-chain at $\sim 56 \mathrm{kDa}$, and 3) the $\gamma$-chain at $\sim 47 \mathrm{kDa}$. SDS PAGE of fibrinogen control approximately confirms molecular weights of the three chains (Figure S4A, lane 2 from left). These three chains are connected by a dimeric disulfide knot (DSK) at the N-termini and many other disulfide bonds along the length of fibrinogen ${ }^{59,60}$. Treatment of $1 \mathrm{mg} / \mathrm{mL}$ of fibrinogen with $0.1 \mathrm{mg} / \mathrm{mL}$ of active MMP 1 at $37^{\circ} \mathrm{C}$ for $24 \mathrm{~h}$ resulted in cleavage of all three chains and a final prominent fragment at $\sim 40 \mathrm{kDa}$ (Figure $\mathbf{S 4 A}$, lane 3 from left). MMP1 completely degraded the $\alpha$-chain and partially degraded the $\beta$-chain within $2 \mathrm{~h}$; however, the $\gamma$-chain took nearly $6 \mathrm{~h}$ to be degraded (Figure S4B). After $8 \mathrm{~h}$, a prominent band at $\sim 40 \mathrm{kDa}$ remained. In other words, the computationally determined sequence of degradation in the open conformation agrees with the experimentally observed sequence (Figure S4B). The agreement between the computational and experimental sequence of degradation further confirms that the open conformations observed in smFRET experiments are functionally relevant.

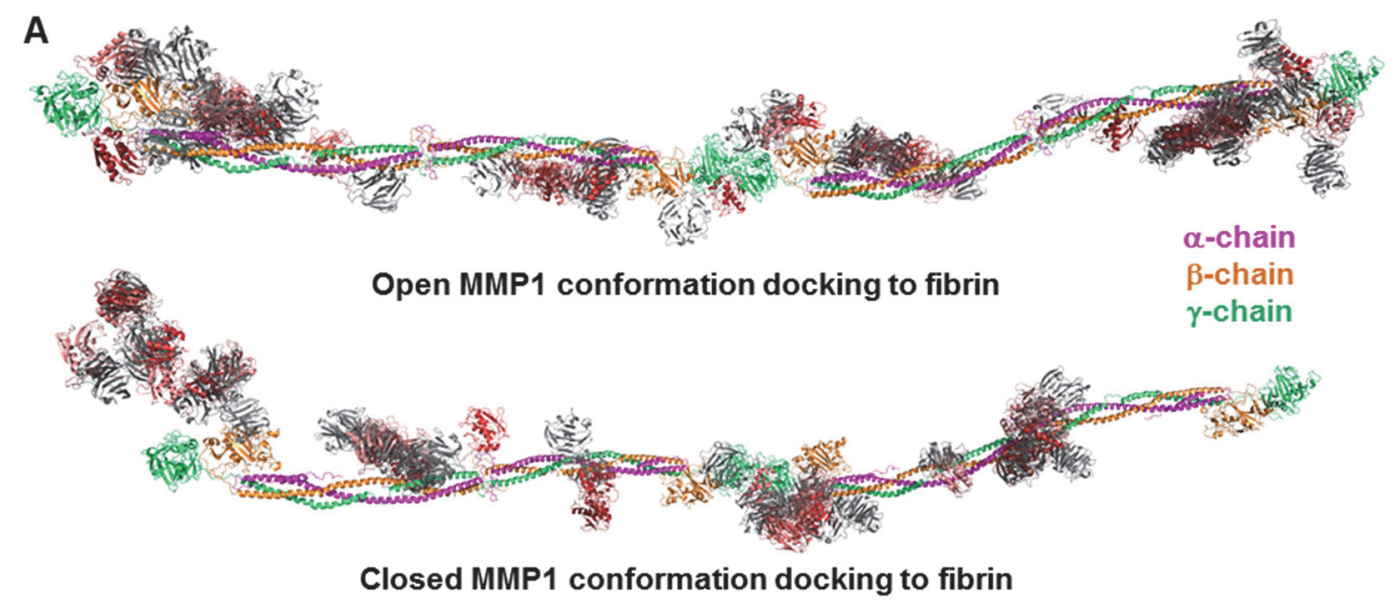

B
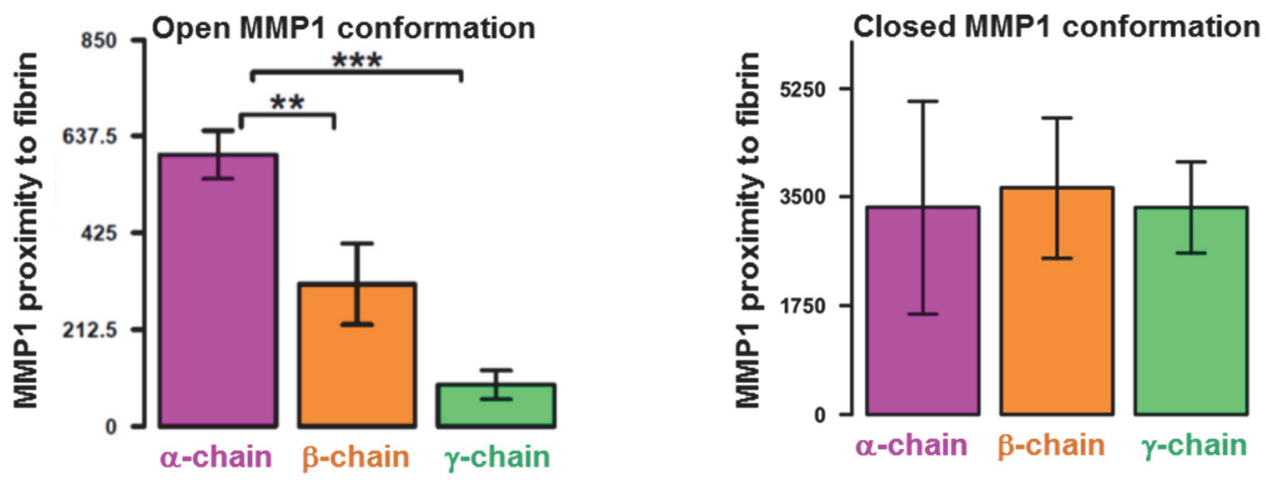

Figure 7. Proximity of MMP1 to the chains of reconstructed fibrin model. (A) The clustered 30 docking poses obtained from ClusPro using fibrin as the ligand and MMP1 as the receptor. The catalytic domain of MMP1 is red, whereas the hemopexin domain is gray. (B) We measured the distance between all possible pairs of atoms between the fibrin chains and MMP1 for 30 docking poses obtained from ClusPro. We counted any distance less than $5 \AA$ and plotted the distributions of total count (y-axis) for each chain for the open and closed conformations. p-value $<0.05:^{*} ;$ p-value $<0.01:^{* *} ; \mathrm{p}$-value $<0.001$ : $^{* *}$. 
We also imaged fibrin without crosslinks after treatment with MMP1 using SEM (Figure S4C), which shows increased porosity of fibrin for active MMP1 consistent with the degradation. We used fibrin without crosslinks because it is easier to form a thin layer of fibrin on a quartz slide for smFRET measurements, and the crystal structure without crosslinks (PDB ID: 1FZC) is available. However, fibrin with crosslinks is more physiologically relevant (see methods for making fibrin with crosslinks). As such, we quantified the degradation rate of fibrin with crosslinks using a weight-based assay (Figure S5). We also performed gel electrophoresis of degradation product (Figure S6A), imaged the reaction volume to show the degradation within $5 \mathrm{~h}$ (Figure S6B), and 302 imaged the surface morphology using SEM (Figure S6C).
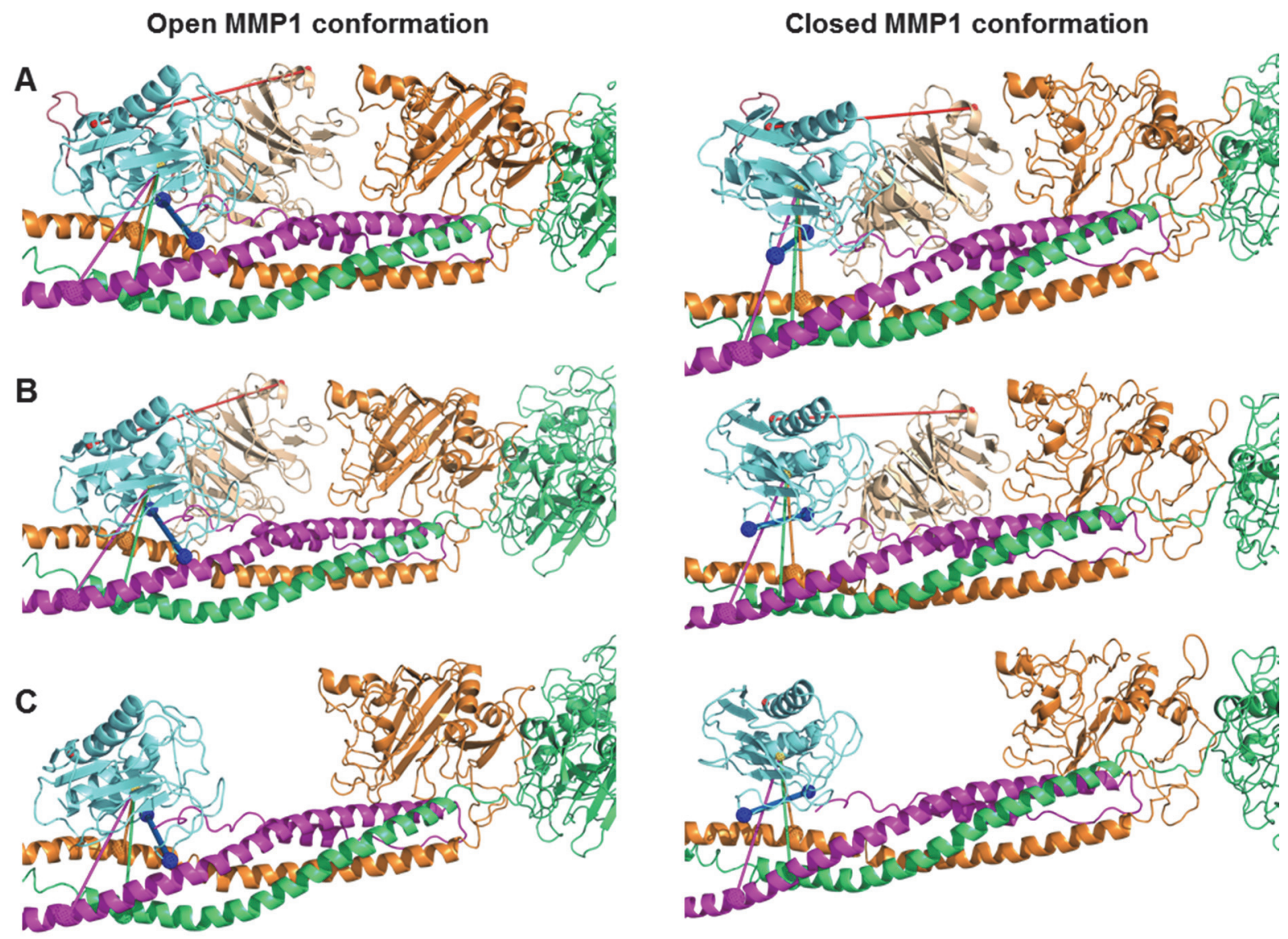

Figure 8. Effects of the linker and reconstructed fibrin on the catalytic pocket opening. The catalytic pocket openings on fibrin represented by the blue line connecting N171 and T230 for (A) the full-length MMP1 (open conformation: $2.68 \pm 0.01 \mathrm{~nm}$; closed conformation: $2.61 \pm 0.01 \mathrm{~nm}$ ); (B) the two MMP1 domains without the linker (open conformation: $2.68 \pm 0.01 \mathrm{~nm}$; closed conformation: $2.61 \pm 0.01 \mathrm{~nm}$ ); and $(\mathbf{C})$ the catalytic domain alone (open conformation: $2.68 \pm 0.01 \mathrm{~nm}$; closed conformation: $2.68 \pm 0.01$ $\mathrm{nm}$ ). The error bars represent the standard deviation of 60 measurements of the catalytic pocket opening obtained from 20 frames each for the three slowest normal modes. ANM simulations of MMP1 interacting with collagen using the crystal structure of MMP1 bound to a collagen model $(4 \mathrm{AUO})^{23}$. We reported that the catalytic and hemopexin domains could communicate via collagen even if the physical linker is removed ${ }^{23}$, which explained the experimental observation that a mixture of the two domains purified separately could degrade 
triple-helical collagen ${ }^{18}$. We wanted to investigate whether or not the two domains communicate via fibrin. We created three forms of MMP1 using the crystal structure (PDB ID: 1SU3): 1) fulllength MMP1, 2) MMP1 with the linker domain removed, and 3) MMP1 with the linker and hemopexin domain removed. We docked both the open and closed conformations with the reconstructed fibrin using ClusPro and chose the binding pose shown in Figure 8. Three forms of MMP1 interacting with fibrin resulted in similar mean and standard deviation of the catalytic pocket opening (Figure 8). In contrast, the catalytic pocket opening on collagen changed even without the physical linker ${ }^{23}$. In other words, fibrin interacts with MMP1 passively, whereas collagen interacts with MMP1 actively. Using single molecule tracking of labeled MMP1 on type1 collagen fibrils, we also showed that fibrils play a role in MMP1 activity due to vulnerable sites on fibrils created during fibril assembly ${ }^{61}$.

Both fibrin and collagen are extracellular and triple-helical but have microstructural and mechanical differences ${ }^{62}$. Fibrin monomers are $45 \mathrm{~nm}$ long and have three right-handed chains forming a left-handed structure in between two globular regions ${ }^{54}$. In contrast, collagen monomers are $300 \mathrm{~nm}$ long and have three left-handed chains forming a right-handed structure ${ }^{63}$. The difference in handedness between fibrin and collagen may lead to in-phase and out-of-phase motion of MMP1 domains. To understand why fibrin and collagen interact differently, however, we need further studies since fibrin and collagen have different sequences and structures despite the triple-helical similarities.

In summary, we have shown that the inter-domain dynamics of MMP1 on fibrin correlates with its fibrinolytic activity. We measured smFRET between two fluorophores attached to the catalytic and hemopexin domains of MMP1. A comparison of distributions of smFRET values between active MMP1 and active site mutant of MMP1 suggests that MMP1 needs to have the two domains well-separated for function. To investigate the roles of open conformations, we measured smFRET in the presence of MMP9 and tetracycline. MMP9 is an enhancer, whereas tetracycline is an inhibitor of MMP1 activity on collagen and a synthetic substrate. In the presence of tetracycline, the inter-domain distance on fibrin becomes smaller consistent with the trend on collagen. MMP9 causes the inter-domain distance of MMP1 to be shorter on fibrin, which is opposite to the observation on collagen. One possible reason is that MMP9 by itself can degrade fibrin but cannot degrade triple-helical collagen. As a result, fibrin degradation by MMP1 in the presence of MMP9 is more complicated. A two-state Poisson process quantitatively describes the inter-domain dynamics. We fitted the histograms of smFRET values to a sum of two Gaussians. The best-fit parameters for the centers are the two states used for simulations. The ratio of kinetic rates equals to the ratio of areas of the two Gaussians fitted to a histogram. Additionally, the sum of kinetic rates equals to the decay rate of autocorrelations of smFRET values. We simulated smFRET trajectories with experimentally determined parameters and noise levels. We recovered the underlying parameters to validate our analyses. The presence of noise in the signal appears to convert a power law autocorrelations into an exponential one.

We performed molecular docking of the open and closed conformations of MMP1 with fibrin. In the closed conformation, MMP1 approaches the three chains equally. In the open conformation, however, MMP1 approaches the $\alpha$-chain first and the $\gamma$-chain last, which we confirmed using SDS PAGE of fibrinogen degraded by MMP1. As such, the open conformation appears to be more functionally relevant. One or both domains of MMP1 binds to fibrin at many places along the length of fibrin. However, the primary location on fibrin is near the globular regions where both the domains bind. We, therefore, selected the docking pose near the globular part for ANM 
simulations. ANM simulations of MMP1-fibrin interactions revealed that the open conformation with well-separated domains has a larger catalytic pocket opening. A larger opening enables the fibrin chains to approach closer to the MMP1 active site. In the closed conformation, the fibrin chains get closer to the catalytic site only when the catalytic domain is alone. We performed limited digestion of fibrin by MMP1 with and without crosslinks and observed increased porosity due to degradation. Also, MMP1 degrades and dissolves crosslinked fibrin within $5 \mathrm{hr}$, suggesting a role of MMP1 in the fibrinolytic pathway. We quantified the degradation using a weight-based assay because interactions of water-soluble MMP1 with water-insoluble fibrin are challenging to quantify using solution-based biochemical assays.

We discussed the results of the MMP1-fibrin system in light of our previous studies on the MMP1collagen system. For both fibrin and collagen, the open conformation of MMP1 is functionally relevant and responds to tetracycline similarly. The open conformation enables larger catalytic pocket opening and closer proximity to the three strands of fibrin and collagen. A two-state Poisson process describes MMP1 dynamics on both fibrin and collagen. However, MMP1 dynamics are opposite to each other on fibrin and collagen in the presence of MMP9. Note that MMP9 can degrade fibrin but cannot degrade triple-helical collagen and, as such, can influence MMP1 dynamics differently. Collagen plays an active role in mediating allosteric communications between the two domains and opening the catalytic pocket. In contrast, fibrin plays a passive role and cannot mediate communications between the two domains. Further studies are needed to investigate the detailed mechanisms of different MMP1 interactions on fibrin and collagen, which may enable allosteric control of MMP1 activity in a substrate-dependent manner for exploring MMPs as drug targets.

\section{Methods}

Purification and labeling of MMP1. Full-length recombinant MMPs were expressed in E. coli and activated using $0.1 \mathrm{mg} / \mathrm{mL}$ trypsin. Activated MMP1 and trypsin together create a chain reaction of further MMP1 activation and broad-spectrum proteolytic cleavage of $E$. coli proteins. Molecular weights of activated MMP1 and trypsin are $\sim 43 \mathrm{kDa}$ and $\sim 23 \mathrm{kDa}$, respectively. We used a $30 \mathrm{kDa}$ cut-off Amicon filter to centrifuge and purify MMP1 in the activated form. For further details, see the previous publication on protease-based purification method ${ }^{14}$. We labeled purified MMP1 with AlexaFluor555 (ThermoFisher Scientific, Cat\# A20346, donor dye) and AlexaFluor647 (ThermoFisher Scientific, Cat\# A20347, acceptor dye) in a ratio of $1: 1$ using maleimide chemistry. $1 \mathrm{~mL}$ of MMP1 at $1 \mathrm{mg} / \mathrm{mL}$ concentration was incubated with $20 \mu \mathrm{L}$ of 1 $\mathrm{mg} / \mathrm{mL}$ AlexaFluor555 and AlexaFluor647 for $1 \mathrm{~h}$ in a $5 \mathrm{~mL}$ glass vial in a nitrogen environment to avoid oxidation of the dyes. After incubation, we used a $30 \mathrm{kDa}$ cut-off Amicon filter to remove free dyes from the solution. We compared the specific activities of labeled and unlabeled MMP1 on the synthetic substrate, MCA-Lys-Pro-Leu-Gly-Leu-DPA-Ala-Arg-NH2, (R\&D Systems, Cat\# ES010) as described before ${ }^{14}$. Labeling MMP1 does not affect its specific activity ${ }^{23}$.

Single-molecule measurements. We prepared $200 \mu \mathrm{L}$ of reaction volume in $10 \mathrm{mM}$ Phosphate Buffer Saline (PBS) (Sigma, Cat\# P3813, pH 7.4) by mixing 10 units of thrombin (Cayman chemical, Cat\# 13188) and $50 \mu \mathrm{g}$ of fibrinogen (Cayman chemical, Cat\# 16088). A thin layer of fibrin was created on a quartz slide similar to the blood smear protocol used in diagnostics ${ }^{64}$ and incubated at $37^{\circ} \mathrm{C}$ for $18 \mathrm{~h}$ without shaking. We made a flow cell of thickness $120 \mu \mathrm{m}$ using a piece of double-sided adhesive tape, a clean quartz slide, and a glass coverslip. The quartz slides had two holes to connect the input and output tubes for exchanging buffers and solutions. We 
created an evanescent wave at the interface of the quartz slide and sample in a Total Internal Reflection Fluorescence (TIRF) Microscope as described before ${ }^{65-68}$. We incubated $50 \mu \mathrm{L}$ of 0.1 $\mathrm{mg} / \mathrm{mL}$ labeled MMP1 with $50 \mu \mathrm{L}$ of protein buffer (50 mM Tris, $100 \mathrm{mM} \mathrm{NaCl}, \mathrm{pH} 8.0$ ) and 100 $\mu \mathrm{g} / \mathrm{mL}$ tetracycline for $30 \mathrm{~min}$ at $22^{\circ} \mathrm{C}$. The labeled MMP1 was serially diluted to prepare a working concentration of $\sim 100 \mathrm{pM}$ and flowed into the flow cell. Alexa555 dyes (donor dyes) attached to MMP1 was excited using a $532 \mathrm{~nm}$ laser and imaged using the TIRF microscope at $22^{\circ} \mathrm{C}$ at $100 \mathrm{~ms}$ time resolution. We imaged Alexa647 and Alexa555 emissions using an EMCCD camera (Andor iXon). The two emission channels were superimposed using a pairwise stitching plugin of ImageJ, and the intensities from the two dyes were extracted and analyzed using Matlab.

Reconstruction of the extended fibrin molecule. We superimposed the crystal structure of fibrinogen (PDB ID: $3 \mathrm{GHG})^{69}$ with the crystal structure of D-D fibrin fragment (PDB ID: 1FZC) ${ }^{70}$ using the Combinatorial Extension (CE) algorithm ${ }^{71}$ implemented in PyMOL ${ }^{72}$ to obtain the reconstructed model of fibrin. The fibrinogen asymmetric unit presents two replicas (either the chains A-F+M-P or G-L+Q-T). Each replica has two ends, which are represented by chains A-C and chains D-F in one and by chains G-I and J-L in the other. Each of such fibrinogen ends could be superimposed to any of the ends of the fibrin crystal (either the chains A-C or chains D-F), yielding a total of 16 different possibilities to reconstruct the fibrin molecule. For each of the superimposed assembly, we superimposed a new fibrin crystal to the fibrinogen biological assembly not used for the initial superimposition. Thus, we superimposed two extra fibrin crystals ( $\mathrm{Fa}$ and $\mathrm{Fb}$, one per superimposed fibrinogen biological assembly) on each of the 16 different reconstruction attempts. A reconstruction compatible with the original fibrinogen packing would show no differences in the positioning of $\mathrm{Fa}$ and $\mathrm{Fb}$. Thus, to select the best superimposition combination to reconstruct the fibrin molecule, the one that minimized the RMSD in between $\mathrm{Fa}$ and $\mathrm{Fb}$ was selected.

Selection of closed and open conformations of MMP1. The activation peptide (residues 32 to 98) was removed from the first biological assembly (chain A) of the crystal structure of MMP1 (PDB ID: 1SU3), leaving only the catalytic (residue numbers 107 to 260), linker (261-277) and hemopexin (278-466) domains of the enzyme, which we selected as the closed conformation. To obtain the open conformation, we submitted the coordinates of the closed conformation to the ANM web server $2.1^{56}$ using default parameters. We extracted the coordinates of the threedimensional fluctuations for 20 frames of the 20 slowest modes. For each frame on each model, we calculated distances using PyMOL. We used the distance between S142 and S366 to analyze the fluctuations between the catalytic and hemopexin domains. We selected the frame that maximized this distance (second mode, second frame) as the MMP1 open conformation.

Molecular docking of MMP1 with fibrin. The closed and open conformations of MMP1 were docked individually with the reconstructed fibrin molecule using the default parameters of ClusPro 2.0 web server ${ }^{57}$. We used the reconstructed fibrin as the receptor and the MMP1 conformations as the ligands. We considered the centroid structure of each of the resulting clusters (maximum 30) as the representative docking solution for a cluster, and we obtained the corresponding weighted scores for the best coefficient for each binding pose. We adjusted different poses to the same Cartesian space by aligning the reconstructed fibrin from each pose to a template one using PyMOL. To analyze the accessibility of MMP1 to the three chains, we counted the number of

441 Preparation of fibrin without and with crosslinks. We prepared fibrin without crosslinks by times an atom in MMP1 was within 5 Angstrom from an atom in the chains. adding 10 units of thrombin (Cayman chemical, Cat\# 13188) to $50 \mu \mathrm{g}$ of fibrinogen (Cayman 
chemical, Cat\# 16088) in $10 \mathrm{mM}$ PBS to a total reaction volume of $200 \mu \mathrm{L}$ and incubating at $37^{\circ}$ $\mathrm{C}$ for $18 \mathrm{~h}$ with $250 \mathrm{rpm}$ shaking inside an incubator (Thermo Fisher Scientific, MaxQ600). Preparation of crosslinked fibrin involved two additional reagents: factor XIII (Abcam, Cat \# ab62427) and $\mathrm{CaCl}_{2}$. Thrombin activates factor XIII to factor XIIIa, which in the presence of $\mathrm{Ca}^{++}$ induces crosslinks in fibrin. We added 10 units of thrombin to $2.5 \mu \mathrm{g}$ of factor XIII in a $0.5 \mathrm{~mL}$ PCR tube, diluted with $10 \mathrm{mM}$ PBS to a total reaction volume of $100 \mu \mathrm{L}$, and incubated at $37^{\circ} \mathrm{C}$ for $10 \mathrm{~min}$. After incubation, we added $50 \mu \mathrm{g}$ of fibrinogen and $10 \mu \mathrm{L}$ of $5 \mathrm{mM} \mathrm{CaCl}_{2}$, diluted with PBS buffer to a total reaction volume of $200 \mu \mathrm{L}$, and incubated at $37^{\circ} \mathrm{C}$ for 15 min to obtain gel-like crosslinked fibrin. Note that fibrin without crosslinks requires a longer incubation time and shaking, but fibrin with crosslinks forms within 15 min without shaking. For every reaction, we put a part of the sample on a sample mount before drying and imaging the surface morphology of fibrin using a Phenom Pro Scanning Electron microscope.

Sodium Dodecyl Sulphate-Polyacrylamide Gel Electrophoresis (SDS PAGE). The degradation profile after fibrinolysis was analyzed using SDS-PAGE. We mixed SDS PAGE loading buffer containing BME (Biorad, Cat\# 610737) with each sample in a 1:1 (v/v) ratio and incubated at $95^{\circ}$ $\mathrm{C}$ for $10 \mathrm{~min}$. We loaded the samples onto the wells of $8 \%$ Tris.glycine gels. We ran electrophoresis in Tris.glycine buffer (Biorad, Cat\# 1610732) for $30 \mathrm{~min}$ at $50 \mathrm{~V}$, followed by $1 \mathrm{~h}$ at $120 \mathrm{~V}$. We used the precision plus protein kaleidoscope pre-stained protein standards (Biorad, Cat\# 1610375) as molecular weight markers. The SDS-PAGE gel was stained with Coomassie Brilliant Blue R250 (Biorad, Cat\# 161-0436) and imaged using a digital camera after destaining.

\section{Acknowledgments}

One grant to S.K.S. and J.K.S. from the National Institutes of Health (RGM137295A) partially supported this work. This project was also partially funded by the professional development funds given to S.K.S and J.K.S. at the Colorado School of Mines. JPI was supported by the grant MSCAfellow@MUNI.

\section{Author contributions}

S.K.S. conceived and designed the overall project. L.K. and S.K.S. designed experiments, L.K. performed experiments, J.P. performed docking and ANM analysis. L.K., J.P., J.K., C.H., J.K., D.W., and S.K.S. analyzed data. S.K.S. wrote the manuscript. All authors edited the manuscript.

\section{Competing financial interests}

The authors declare no competing financial interests.

\section{Data availability}

The datasets generated during and/or analyzed during the current study are available from the corresponding author on reasonable request. 


\section{References}

4831 Karplus, M. \& Kuriyan, J. Molecular dynamics and protein function. Proceedings of the National Academy of Sciences 102, 6679-6685 (2005).

2 Henzler-Wildman, K. \& Kern, D. Dynamic personalities of proteins. Nature 450, 964-972 (2007).

4873 Henzler-Wildman, K. A. et al. A hierarchy of timescales in protein dynamics is linked to enzyme catalysis. Nature 450, 913-916 (2007).

4 Lewandowski, J. R., Halse, M. E., Blackledge, M. \& Emsley, L. Direct observation of hierarchical protein dynamics. Science 348, 578-581 (2015).

5 Bhaskara, R. M., de Brevern, A. G. \& Srinivasan, N. Understanding the role of domaindomain linkers in the spatial orientation of domains in multi-domain proteins. Journal of Biomolecular Structure and Dynamics 31, 1467-1480 (2013). Esteban-Martín, S. et al. Correlated inter-domain motions in adenylate kinase. PLoS computational biology 10 (2014). Ratnikov, B. I. et al. Basis for substrate recognition and distinction by matrix

23223-23232 (2016). metalloproteinases. Proceedings of the National Academy of Sciences 111, E4148-E4155 (2014).

8 Piccard, H., Van den Steen, P. E. \& Opdenakker, G. Hemopexin domains as multifunctional liganding modules in matrix metalloproteinases and other proteins. Journal of leukocyte biology 81, 870-892 (2007).

10 Rodríguez, D., Morrison, C. J. \& Overall, C. M. Matrix metalloproteinases: what do they not do? New substrates and biological roles identified by murine models and proteomics. Biochimica et Biophysica Acta (BBA)-Molecular Cell Research 1803, 39-54 (2010).

11 Page-McCaw, A., Ewald, A. J. \& Werb, Z. Matrix metalloproteinases and the regulation of tissue remodelling. Nature reviews. Molecular cell biology 8, 221-233, doi:10.1038/nrm2125 (2007).

12 Jackson, B. C., Nebert, D. W. \& Vasiliou, V. Update of human and mouse matrix metalloproteinase families. Human genomics 4, 194 (2010).

13 Manka, S. W. et al. Structural insights into triple-helical collagen cleavage by matrix metalloproteinase 1. Proceedings of the National Academy of Sciences 109, 12461-12466 (2012).

14 Kumar, L., Colomb, W., Czerski, J., Cox, C. R. \& Sarkar, S. K. Efficient protease based purification of recombinant matrix metalloprotease-1 in E. coli. Protein expression and purification 148, 59-67 (2018). of Matrix Metalloproteinase-1 · Triple-Helical Peptide Complexes. The Journal of Physical Chemistry B, 5316-5326 (2017).

16 Fields, G. B. Interstitial collagen catabolism. $J$ Biol Chem 288, 8785-8793, doi:10.1074/jbc.R113.451211 (2013).

17 Bertini, I. et al. Structural basis for matrix metalloproteinase 1-catalyzed collagenolysis. Journal of the American Chemical Society 134, 2100-2110 (2012). 
52618 Chung, L. D. et al. Collagenase unwinds triple-helical collagen prior to peptide bond hydrolysis. Embo Journal 23, 3020-3030 (2004).

19 Lauer-Fields, J. L., Juska, D. \& Fields, G. B. Matrix metalloproteinases and collagen catabolism. Biopolymers 66, 19-32 (2002).

20 Salsas-Escat, R., Nerenberg, P. S. \& Stultz, C. M. Cleavage site specificity and conformational selection in type I collagen degradation. Biochemistry 49, 4147-4158 (2010).

21 Nerenberg, P. S. \& Stultz, C. M. Differential unfolding of alpha 1 and alpha 2 chains in type I collagen and collagenolysis. Journal of Molecular Biology 382, 246-256 (2008). Nerenberg, P. S., Salsas-Escat, R. \& Stultz, C. M. Do collagenases unwind triple-helical collagen before peptide bond hydrolysis? Reinterpreting experimental observations with mathematical models. Proteins: Structure, Function, and Bioinformatics 70, 1154-1161 (2008).

23 Kumar, L. et al. Allosteric communications between domains modulate the activity of matrix metalloprotease-1. bioRxiv, 804559, doi:10.1101/804559 (2020).

Heit, J. A., Cohen, A. T. \& Anderson, F. A. Estimated annual number of incident and recurrent, non-fatal and fatal venous thromboembolism (VTE) events in the US. (2005). Mahan, C. E. et al. Venous thromboembolism: annualised United States models for total, hospital-acquired and preventable costs utilising long-term attack rates. Thrombosis and haemostasis 107, 291-302 (2012).

26 Deitelzweig, S., Johnson, B., Lin, J. \& Schulman, K. Prevalence of clinical venous thromboembolism in the USA: current trends and future projections. American journal of hematology 86, 217-220 (2011).

27 Collen, D. The plasminogen (fibrinolytic) system. Thrombosis and haemostasis 82, 259270 (1999).

28 dela Peña, I., Borlongan, C., Shen, G. \& Davis, W. Strategies to extend thrombolytic time window for ischemic stroke treatment: an unmet clinical need. Journal of stroke 19, 50 (2017).

29 Rijken, D. \& Lijnen, H. New insights into the molecular mechanisms of the fibrinolytic system. Journal of thrombosis and haemostasis 7, 4-13 (2009).

30 Agnelli, G., Buchanan, M. R., Fernandez, F. \& Hirsh, J. The thrombolytic and hemorrhagic effects of tissue type plasminogen activator: Influence of dosage regimens in rabrits. Thrombosis research 40, 769-777 (1985).

31 Ohbayashi, T. et al. Degradation of fibrinogen and collagen by staphopains, cysteine proteases released from Staphylococcus aureus. Microbiology 157, 786-792 (2011).

32 Lund, L. R. et al. Functional overlap between two classes of matrix-degrading proteases in wound healing. The EMBO Journal 18, 4645-4656 (1999).

33 Saito, S. et al. Role of matrix metalloproteinases 1, 2, and 9 and tissue inhibitor of matrix metalloproteinase-1 in chronic venous insufficiency. Journal of Vascular Surgery 34, 930937 (2001).

34 Asahi, M. et al. Role for matrix metalloproteinase 9 after focal cerebral ischemia, effects of gene knockout and enzyme inhibition with BB-94. Journal of Cerebral Blood Flow and Metabolism 20, 1681-1689 (2000). 
57236 Koshiba, T. et al. Detection of matrix metalloproteinase activity in human pancreatic cancer. Surgery Today-the Japanese Journal of Surgery 27, 302-304 (1997).

37 Hanemaaijer, R., Visser, H., Konttinen, Y. T., Koolwijk, P. \& Verheijen, J. H. A novel and simple immunocapture assay for determination of gelatinase-B (MMP-9) activities in biological fluids: Saliva from patients with Sjogren's syndrome contain increased latent and active gelatinase-B levels. Matrix Biology 17, 657-665 (1998).

38 Sakalihasan, N., Delvenne, P., Nusgens, B. V., Limet, R. \& Lapiere, C. M. Activated forms of MMP(2) and MMP(9) in abdominal aortic aneurysms. Journal of Vascular Surgery 24,

39 Bini, A., Itoh, Y., Kudryk, B. J. \& Nagase, H. Degradation of Cross-Linked Fibrin by Matrix Metalloproteinase 3 (Stromelysin 1): Hydrolysis of the $\gamma$ Gly 404- Ala 405 Peptide Bond. Biochemistry 35, 13056-13063 (1996).

40 Lelongt, B. et al. Matrix Metalloproteinase 9 Protects Mice from Anti-Glomerular Basement Membrane Nephritis through Its Fibrinolytic Activity. Journal of Experimental Medicine 193, 793-802 (2001).

41 Hotary, K. B. et al. Matrix metalloproteinases (MMPs) regulate fibrin-invasive activity via MT1-MMP-dependent and-independent processes. Journal of Experimental Medicine 195, 295-308 (2002).

42 Lijnen, H. Matrix metalloproteinases and cellular fibrinolytic activity. Biochemistry (Moscow) 67, 92-98 (2002).

43 Merchant, K. A., Best, R. B., Louis, J. M., Gopich, I. V. \& Eaton, W. A. Characterizing the unfolded states of proteins using single-molecule FRET spectroscopy and molecular simulations. Proceedings of the National Academy of Sciences 104, 1528-1533 (2007). Best, R. B. et al. Effect of flexibility and cis residues in single-molecule FRET studies of polyproline. Proceedings of the National Academy of Sciences 104, 18964-18969 (2007). Stennett, E. M., Ciuba, M. A. \& Levitus, M. Photophysical processes in single molecule organic fluorescent probes. Chemical Society Reviews 43, 1057-1075 (2014). antibleaching reagent. Journal of the American Chemical Society 131, 5018-5019 (2009). Rasnik, I., McKinney, S. A. \& Ha, T. Nonblinking
fluorescence imaging. Nature methods 3, 891 (2006). Kochevar, I. E. \& Redmond, R. W. in Methods in enzymology Vol. 319 20-28 (Elsevier, 2000). polymethine fluorescent dyes in biomolecular environments. Quarterly reviews of biophysics 44, 123-151 (2011).

50 Kumar, L. et al. Allosteric communications between domains modulate activity of matrix metalloprotease-1. bioRxiv, 804559 (2019).

51 Zhuang, X. et al. A single-molecule study of RNA catalysis and folding. Science 288, 2048-2051 (2000).

52 Jarymowycz, V. A. \& Stone, M. J. Fast time scale dynamics of protein backbones: NMR relaxation methods, applications, and functional consequences. Chemical reviews 106, 1624-1671 (2006).

53 Wertheim, G., Butler, M., West, K. \& Buchanan, D. Determination of the Gaussian and Lorentzian content of experimental line shapes. Review of Scientific Instruments 45, 13691371 (1974). 
54 Weisel, J. W. \& Litvinov, R. I. in Fibrous proteins: structures and mechanisms

$405-456$ (Springer, 2017).

Shoulders, M. D. \& Raines, R. T. Collagen structure and stability. Annual review of biochemistry 78, 929-958 (2009).

56 Eyal, E., Lum, G. \& Bahar, I. The anisotropic network model web server at 2015 (ANM 2.0). Bioinformatics 31, 1487-1489 (2015).

57 Kozakov, D. et al. The ClusPro web server for protein-protein docking. Nature protocols 12, 255-278 (2017).

58 Bini, A., Wu, D., Schnuer, J. \& Kudryk, B. J. Characterization of stromelysin 1 (MMP-3), matrilysin (MMP-7), and membrane type 1 matrix metalloproteinase (MT1-MMP) derived fibrin (ogen) fragments D-dimer and D-like monomer: NH2-terminal sequences of latestage digest fragments. Biochemistry 38, 13928-13936 (1999).

59 GÅRDLUND, B., HESSEL, B., BLOMBÄCK, B., MARGUERIE, G. \& MURANO, G. Primary Structure of Human Fibrinogen: Characterization of Disulfide-Containing Cyanogen-Bromide Fragments. European journal of biochemistry 77, 595-610 (1977).

60 Blombäck, B. \& Blombäck, M. The molecular structure of fibrinogen. Annals of the New York Academy of Sciences 202, 77-97 (1972).

61 Dittmore, A. et al. Internal strain drives spontaneous periodic buckling in collagen and regulates remodeling. Proceedings of the National Academy of Sciences, 201523228 (2016).

62 Lai, V. K. et al. Microstructural and mechanical differences between digested collagenfibrin co-gels and pure collagen and fibrin gels. Acta biomaterialia 8, 4031-4042 (2012). Gelse, K., Pöschl, E. \& Aigner, T. Collagens - structure, function, and biosynthesis. Advanced drug delivery reviews 55, 1531-1546 (2003).

64 Bain, B. J. Diagnosis from the blood smear. New England Journal of Medicine 353, 498507 (2005).

65 Dittmore, A. et al. Internal strain drives spontaneous periodic buckling in collagen and regulates remodeling. Proceedings of the National Academy of Sciences, 8436-8441 (2016).

66 Sarkar, S. K., Marmer, B., Goldberg, G. \& Neuman, K. C. Single-molecule tracking of collagenase on native type I collagen fibrils reveals degradation mechanism. Current Biology 22, 1047-1056 (2012).

67 Colomb, W., Czerski, J., Sau, J. \& Sarkar, S. Estimation of microscope drift using fluorescent nanodiamonds as fiducial markers. Journal of Microscopy 266, 298-306 (2017). human fibrinogen. Biochemistry 48, 3877-3886 (2009).

70 Everse, S. J., Spraggon, G., Veerapandian, L., Riley, M. \& Doolittle, R. F. Crystal structure

660 of fragment double-D from human fibrin with two different bound ligands. Biochemistry 37, 8637-8642 (1998).

71 Shindyalov, I. N. \& Bourne, P. E. Protein structure alignment by incremental combinatorial extension (CE) of the optimal path. Protein engineering 11, 739-747 (1998). 
bioRxiv preprint doi: https://doi.org/10.1101/853796; this version posted July 6, 2020. The copyright holder for this preprint (which was not certified by peer review) is the author/funder. All rights reserved. No reuse allowed without permission.

66372 Schrodinger, L. The PyMOL molecular graphics system, version 1.8. Schrodinger LLC, $664 \quad$ New York, NY (2015). 


\section{Supplementary Information}

Corresponding author: 
24 We fitted a sum of two Gaussians to the experimental histograms using the following equation:

$$
y=a_{1} \times e^{-\frac{\left(x-b_{1}\right)^{2}}{c_{1}^{2}}}+a_{2} \times e^{-\frac{\left(x-b_{2}\right)^{2}}{c_{2}^{2}}}
$$

where $\mathrm{a}, \mathrm{b}$, and c are amplitude, center, and width of the Gaussian, respectively. The parameters b1

Table S1. Best-fit parameters for experimental histograms and autocorrelations. (A) A sum of two Gaussians fits the experimental histograms in Figure 3. (B) An exponential distribution fits the experimental autocorrelations in Figure 3. Power law distribution does not fit the experimental autocorrelations. (C) Kinetic rates of interconversion between the two states, S1 and S2, from the histograms and autocorrelations. Yellow highlighted numbers are (A) the centers of Gaussian fits and (B) the decay rates of exponential fits, e. The error bars represent the standard errors of mean.

\section{A Gaussian fit parameters for experimental histograms}

\begin{tabular}{|c|c|c|c|c|c|c|}
\hline \multicolumn{3}{|c|}{ MMP1 without ligands } & \multicolumn{2}{c|}{ MMP1 with MMP9 } & \multicolumn{2}{c|}{ MMP1 with tetracycline } \\
\hline \multicolumn{2}{|c|}{ Active } & Inactive & Active & Inactive & Active & Inactive \\
\hline a1 & $1.99 \pm 0.13$ & $0.90 \pm 0.17$ & $2.14 \pm 0.06$ & $1.18 \pm 3.21$ & $4.38 \pm 0.05$ & $0.60 \pm 0.79$ \\
\hline b1/S1 & $0.42 \pm 0.01$ & $0.52 \pm 0.01$ & $0.46 \pm 0.01$ & $0.46 \pm 0.18$ & $0.61 \pm 0.01$ & $0.49 \pm 0.13$ \\
\hline c1 & $0.08 \pm 0.01$ & $0.10 \pm 0.01$ & $0.11 \pm 0.01$ & $0.11 \pm 0.06$ & $0.10 \pm 0.01$ & $0.11 \pm 0.06$ \\
\hline a2 & $5.52 \pm 0.15$ & $8.12 \pm 0.17$ & $5.58 \pm 0.07$ & $4.61 \pm 4.12$ & $2.10 \pm 0.12$ & $5.6 \pm 1.21$ \\
\hline b2/S2 & $0.51 \pm 0.01$ & $0.55 \pm 0.01$ & $0.51 \pm 0.01$ & $0.53 \pm 0.01$ & $0.70 \pm 0.01$ & $0.58 \pm 0.01$ \\
\hline c2 & $0.07 \pm 0.01$ & $0.06 \pm 0.01$ & $0.06 \pm 0.01$ & $0.09 \pm 0.01$ & $0.06 \pm 0.01$ & $0.09 \pm 0.01$ \\
\hline
\end{tabular}

B Exponential fit parameters for correlations

\begin{tabular}{|c|c|c|c|c|c|c|}
\hline \multicolumn{3}{|c|}{ MMP1 without ligands } & \multicolumn{2}{c|}{ MMP1 with MMP9 } & \multicolumn{2}{c|}{ MMP1 with tetracycline } \\
\hline \multicolumn{2}{|c|}{ Active } & Inactive & Active & Inactive & Active & Inactive \\
\hline d & $0.37 \pm 0.01$ & $0.15 \pm 0.01$ & $0.21 \pm 0.01$ & $0.45 \pm 0.01$ & $0.62 \pm 0.01$ & $0.36 \pm 0.01$ \\
\hline e & $0.08 \pm 0.01$ & $0.07 \pm 0.01$ & $0.09 \pm 0.01$ & $0.05 \pm 0.01$ & $0.05 \pm 0.01$ & $0.06 \pm 0.01$ \\
\hline f & $-0.03 \pm 0.01$ & $-0.01 \pm 0.01$ & $-0.01 \pm 0.01$ & $-0.03 \pm 0.01$ & $-0.05 \pm 0.01$ & $-0.004 \pm 0.001$ \\
\hline
\end{tabular}

\section{Kinetic rates calculated from histograms and correlations}

\begin{tabular}{|c|c|c|c|c|c|c|}
\hline \multicolumn{2}{|c|}{ MMP1 without ligands } & \multicolumn{2}{c|}{ MMP1 with MMP9 } & \multicolumn{2}{c|}{ MMP1 with tetracycline } \\
\hline \multicolumn{2}{|c|}{ Active } & Inactive & Active & Inactive & Active & Inactive \\
\hline $\mathbf{k 1}\left(\mathbf{s}^{-1}\right)$ & 0.0567 & 0.0591 & 0.0528 & 0.0381 & 0.0112 & 0.0531 \\
\hline $\mathbf{k} 2\left(\mathbf{s}^{-1}\right)$ & 0.0233 & 0.0109 & 0.0372 & 0.0119 & 0.0388 & 0.0069 \\
\hline
\end{tabular}

29

30

31

33 where $C_{\tau}$ is the autocorrelation at lag number $\tau, N$ is the number of points in a FRET trajectory,

We subtracted the average FRET value from each FRET trajectory and used the following equation to calculate autocorrelations:

$$
C_{\tau}=\frac{1}{N-\tau} \sum_{t=0}^{N-\tau}\left\{I(t)-\frac{1}{N-\tau} \sum_{t^{\prime}=0}^{N-\tau} I(t)\right\} \times\left\{I(t+\tau)-\frac{1}{N-\tau} \sum_{t^{\prime}=\tau}^{N} I(t)\right\}
$$

34 and $I(t)$ is the FRET value at $t$. 
35 We normalized autocorrelations by dividing autocorrelations at each lag by $C_{\tau=0}$. We fitted autocorrelations between $\tau=1$ and $\tau=1000$ to both power law and exponential distributions. For power law, we used a form of Pareto distribution ${ }^{1}$ that satisfies the boundary conditions of our

38 calculated autocorrelations, i.e., $C_{\tau=0}=1$ at $t=0$ and $C_{\tau=\infty}=0$ at $t=\infty$. We fitted the following equations of power law and exponential functions:

$$
C_{\tau}=(a \times \tau+1)^{-b}
$$

$$
C_{\tau}=d \times \exp ^{-e \times \tau}+f
$$

Best-fit parameters for two-state simulations. We simulated smFRET trajectories assuming that MMP1 undergoes interconversion between two states, S1 and S2, which are the centers of Gaussian fits to the histograms. We considered active MMP1 and active site mutant of MMP1 without ligands (Figure 3A). We simulated $350 \mathrm{smFRET}$ trajectories, each $1000 \mathrm{~s}$ long, with the input parameters in Table S2. We analyzed the simulated and experimental trajectories similarly. The recovered parameters (Table S2, right side) agree well with the input parameters.

Table S2. MMP1 inter-domain dynamics as a two-state Poisson process. Analysis of FRET trajectories for MMP1 without ligands recovered the centers and decay rates used as inputs. The agreement between the input and retrieved values suggests that a two-state Poisson process describes the MMP1 dynamics.

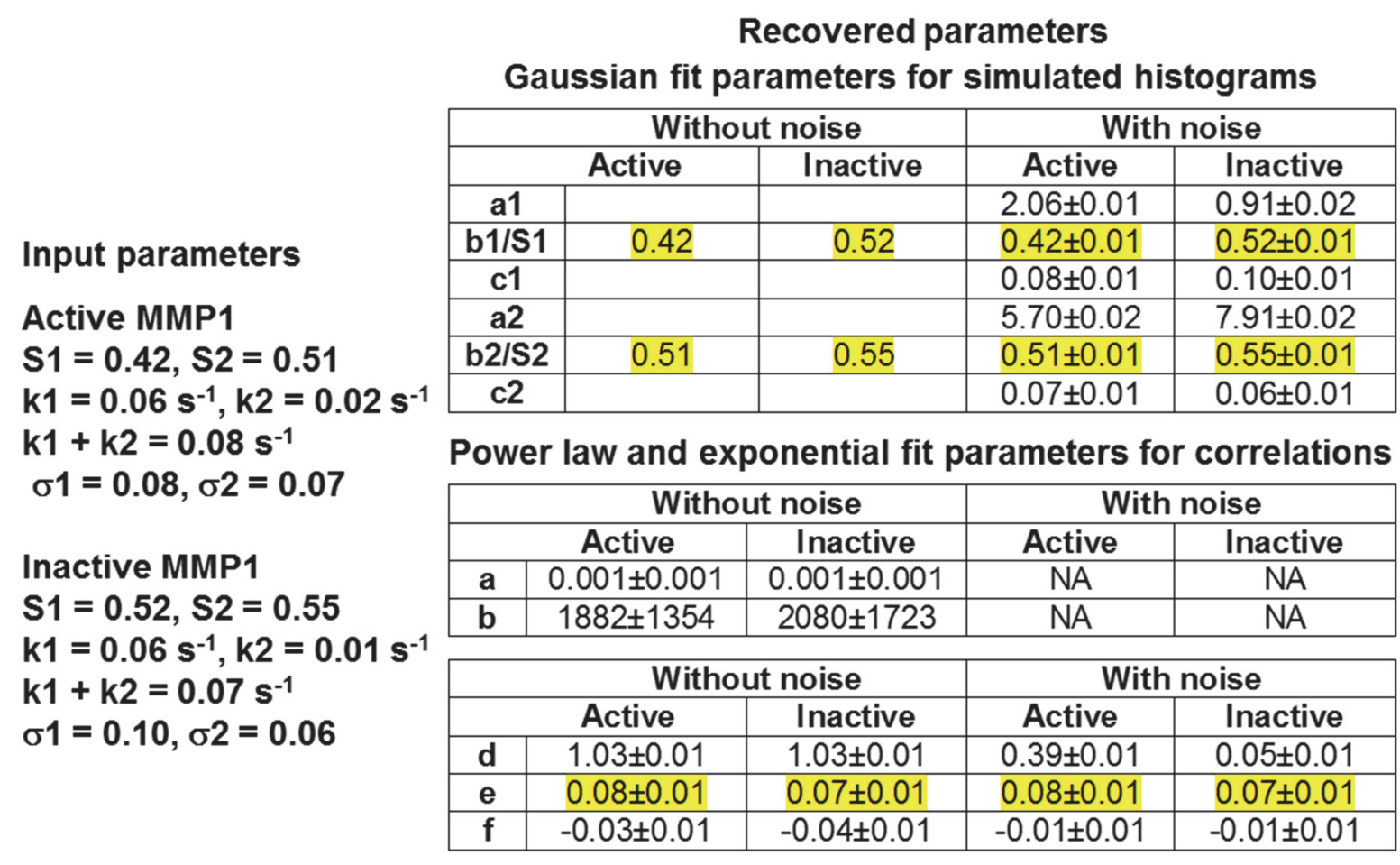



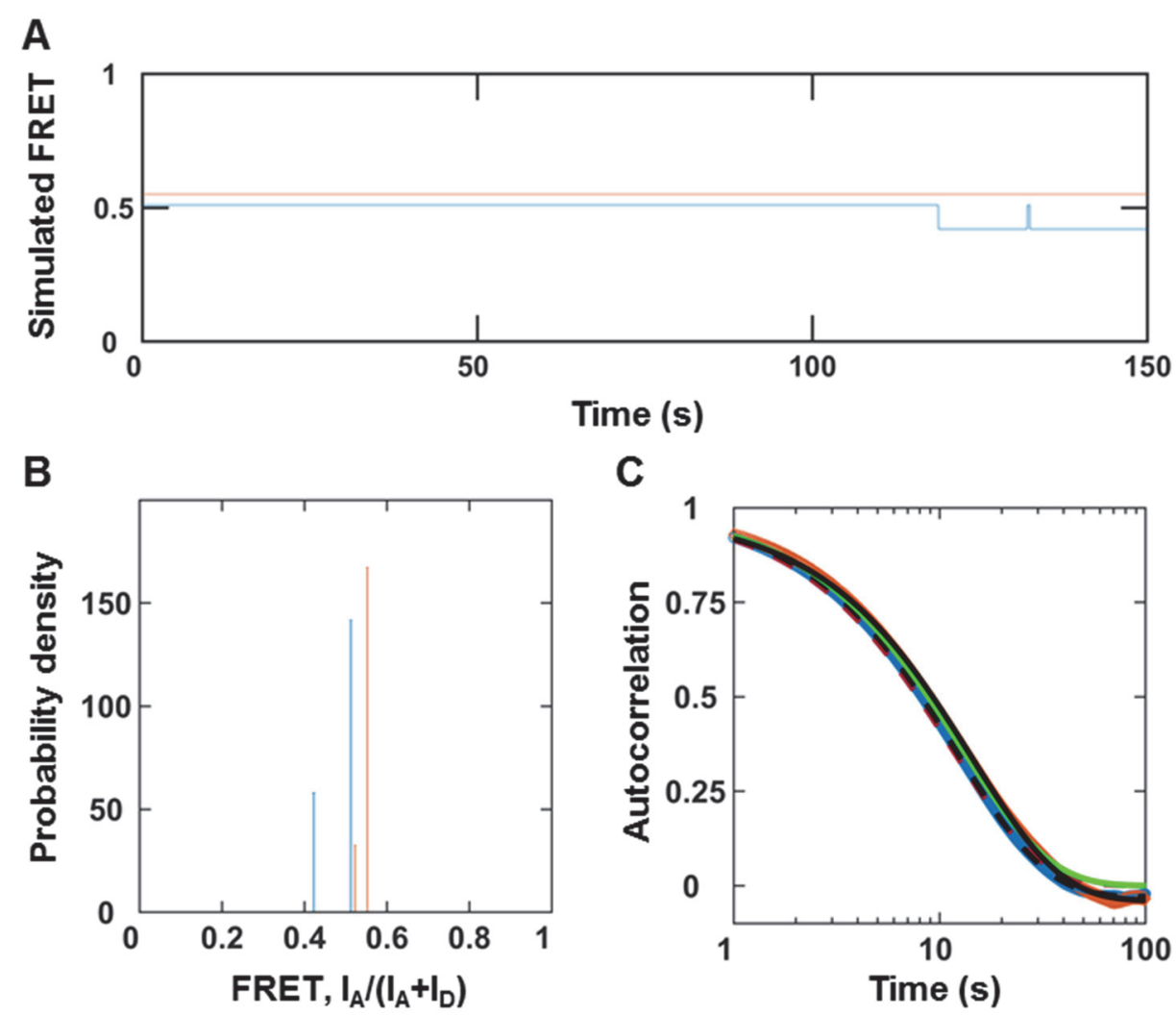

Figure S1. MMP1 inter-domain dynamics as a Poisson process without noise. (A) An example of simulated two-state FRET trajectory without noise for active MMP1 (blue) and active site mutant of MMP1 (orange). (B) Histograms of the recovered FRET values with bin size $=0.005$. (C) Autocorrelations of simulated trajectories recover the sum, $\mathrm{k} 1+\mathrm{k} 2$, from exponential fits (active MMP1: dashed black line; active site mutant of MMP1: solid black line). Note that power law does not fit the autocorrelations with noise (Fig. 4). However, power law fits the autocorrelations without noise (active MMP1: dashed red line; active site mutant of MMP1: solid green line).The error bars are the sems for histograms and autocorrelations and are too small to be seen.

51 Potential sites of interactions between MMP1 and fibrin. MMP3, MMP7, and MMP14 cleave 52 the $\alpha$-chain at Asp97-Phe98 and Asn102-Asn103; the $\beta$-chain at Asp123-Leu124, Asn13753 Val138, and Glu141-Tyr142; and the $\gamma$-chain at Thr83-Leu84 ${ }^{2}$ as shown in Figure S2.

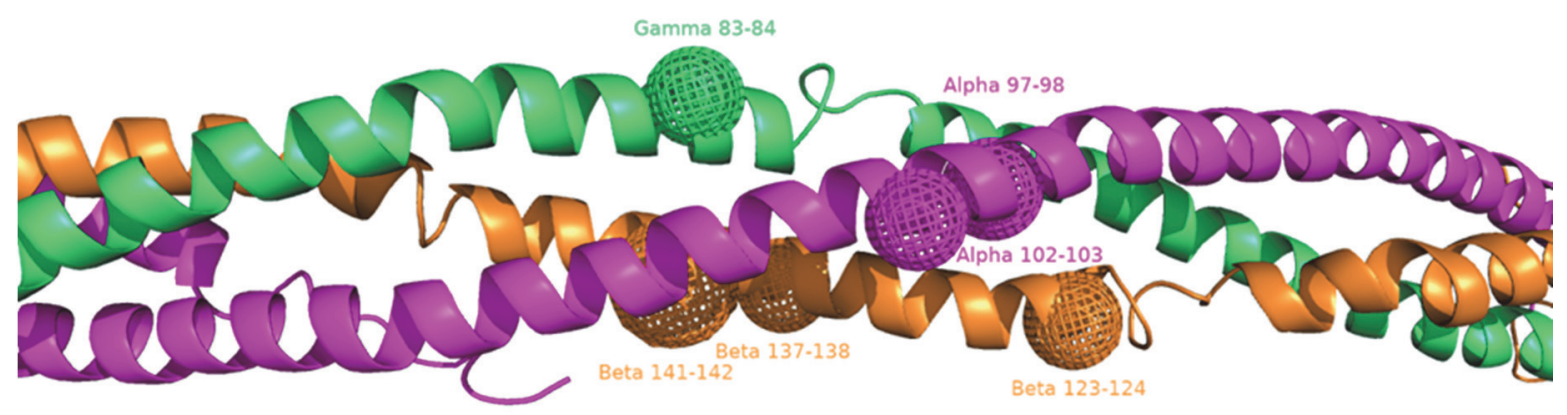

Figure S2. Potential MMP1 cleavage sites on the three fibrin chains. The balls are potential cleavage sites on fibrin that we considered to select the best docking pose between MMP1 and fibrin. 
A

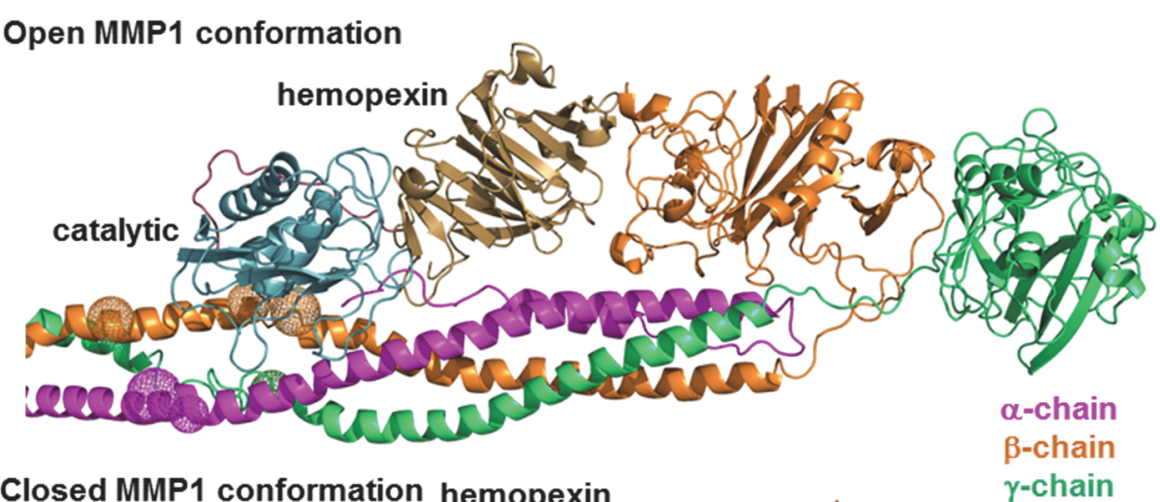

B Closed MMP1 conformation hemopexin $\gamma$-chain
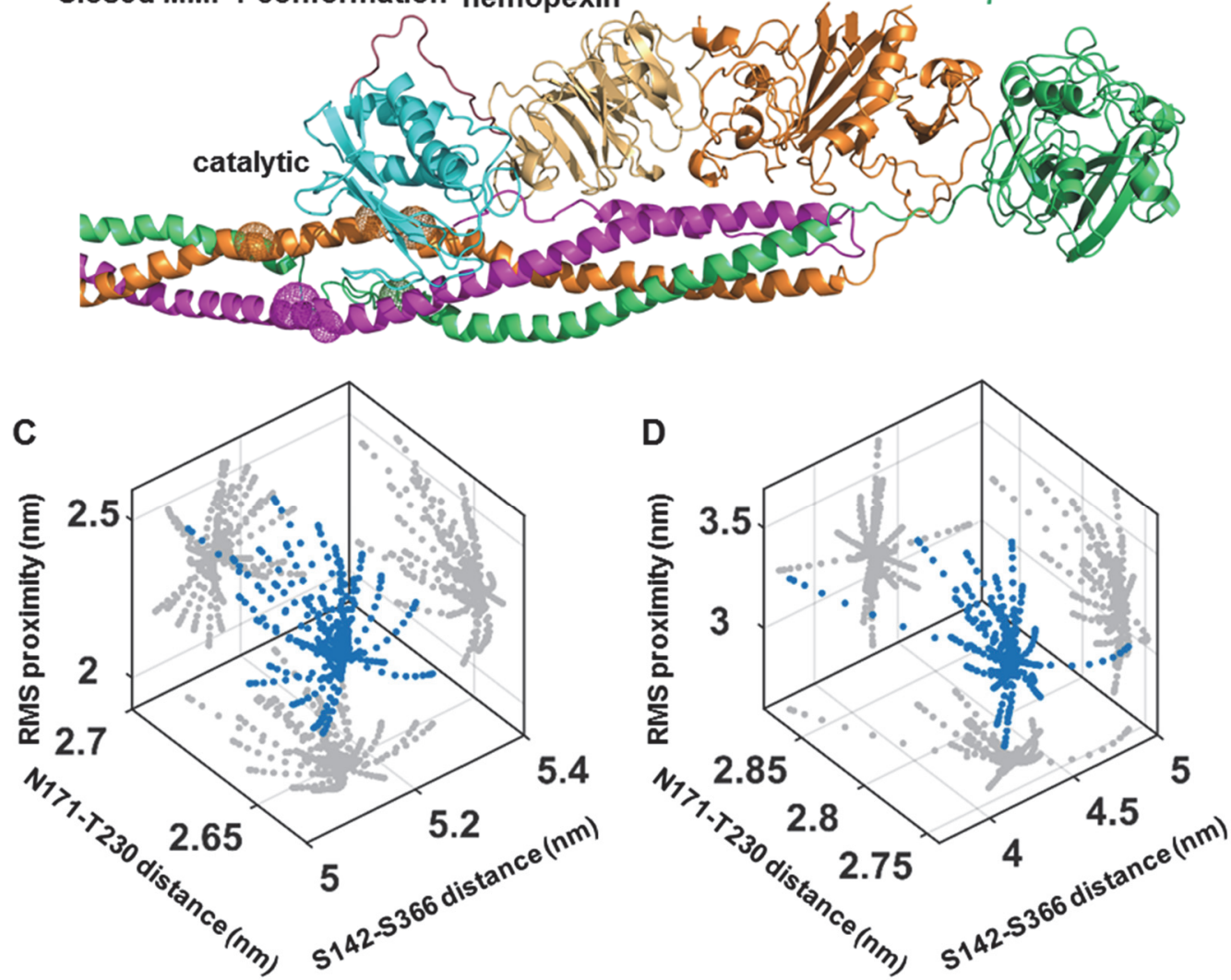

Figure S3. Correlations of MMP1 inter-domain distance with catalytic pocket opening when MMP1 is bound to fibrinogen. Examples of (A) open and (B) closed conformations of MMP1 (PDB ID: 1SU3) bound to fibrinogen (PDB ID: 3GHG). Three dimensional scatter plots (blue circle) of interdomain distance (S142-S366), catalytic pocket opening (N171-T230), and rms proximity between the MMP1 catalytic site and the three fibrinogen chains for $(\mathbf{C})$ open and (D) closed MMP1 conformations. Two-dimensional projections of the scatter plots are in gray.

MMP1 (Figure S3) shows smaller catalytic pocket openings for the open MMP1 conformations. However, fibrinogen-bound MMP1 shows closer proximity to the fibrinogen chains in agreement with free MMP1 and fibrin-bound MMP1. The smaller catalytic pocket opening suggests a different mechanism of fibrinogen and fibrin degradation by MMP1, which needs further studies to confirm. 
MMP1 activity on fibrinogen. The point mutation E219Q renders MMP1 catalytically inactive on collagen. For fibrinogen degradation, however, the E219Q mutant degraded the $\alpha$ - and $\beta$ chains, but did not degrade the $\gamma$-chain (Figure S4A, lane 4 from left). We used trypsin during MMP1 purification, and trypsin is known to degrade fibrinogen ${ }^{3}$; therefore, any residual trypsin could potentially interfere with the results. To address this possibility, we added $0.5 \mathrm{mg} / \mathrm{mL}$ trypsin inhibitor to all the reactions, which was sufficient to inhibit $0.1 \mathrm{mg} / \mathrm{mL}$ trypsin (Figure $\mathbf{S 4 A}$, lane 5 from left).
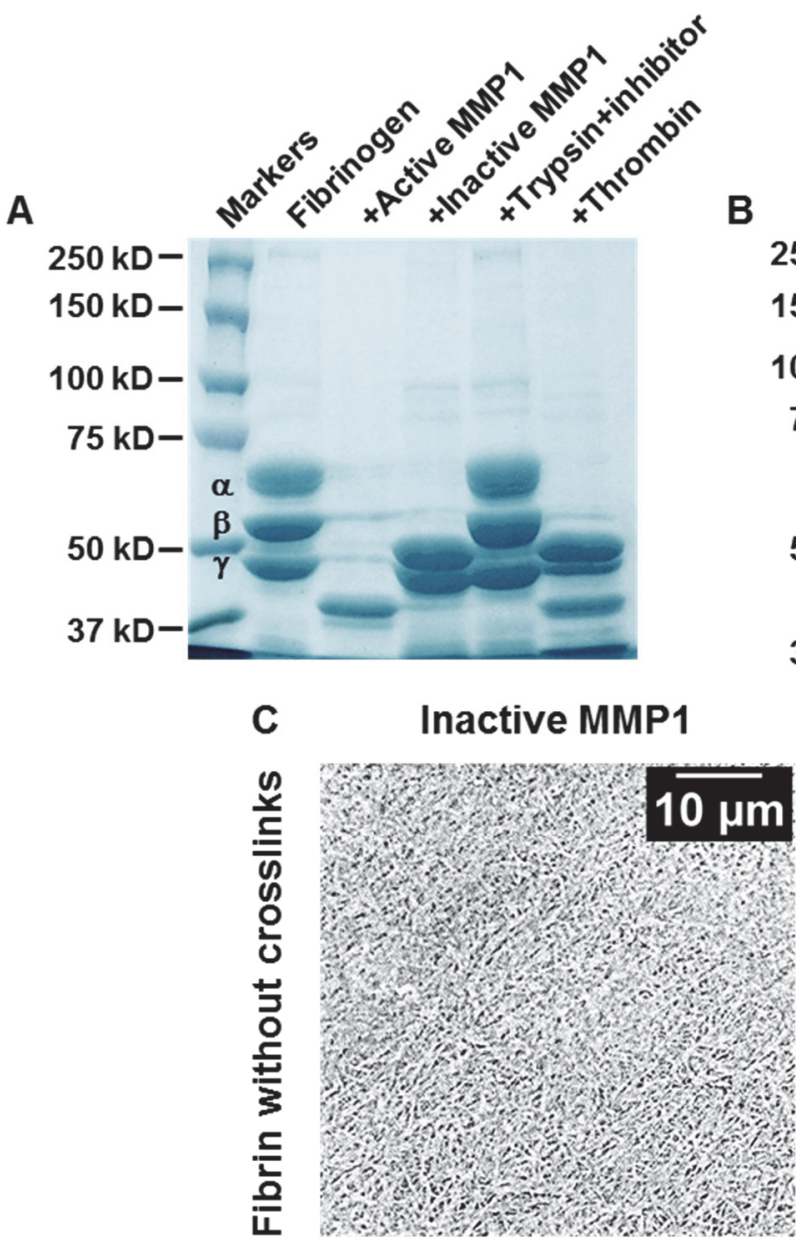

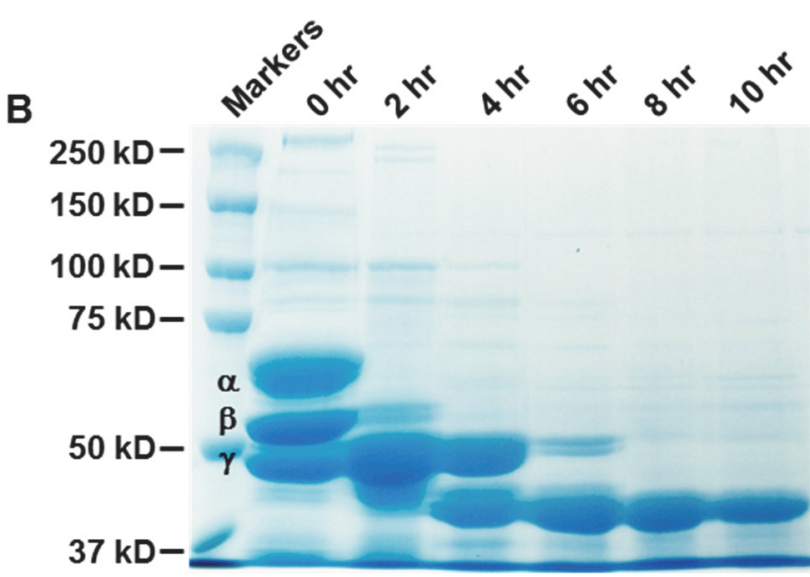

Active MMP1

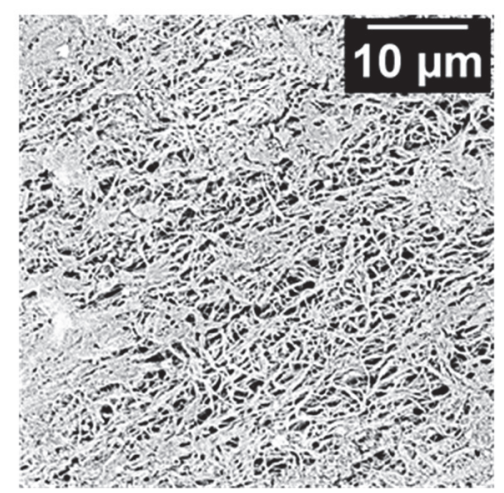

Figure S4. Ensemble activity of MMP1 on fibrinogen and fibrin without crosslinks. (A) SDS PAGE of fibrinogen treated with different enzymes. Since we used trypsin to activate and purify MMP1, we added $0.5 \mathrm{mg} / \mathrm{mL}$ trypsin inhibitor to lane 2-6 to control for any residual effect of trypsin. Trypsin inhibitor (Worthington, Lima Bean, Cat\# LS002829) at $0.5 \mathrm{mg} / \mathrm{mL}$ inhibited the fibrinolytic effect of 0.1 $\mathrm{mg} / \mathrm{mL}$ trypsin used in lane 5 . Note that the difference between trypsin inhibitor and MMP1 inhibitor (tetracycline). (B) Time-dependent fibrinogen degradation by active MMP1. (C) SEM images of fibrin after MMP1 treatment. Note that the active site mutant of MMP1 is catalytically inactive on collagen ${ }^{4}$. However, as evident from Figure S4A, active site mutant of MMP1 is partially active on fibrinogen. In contrast, active site mutant of MMP1 is catalytically inactive on crosslinked fibrin, as shown in Figure S5 and Figure S6.

68 Even though limited digestion of fibrinogen by thrombin leads to fibrin, the longer digestion for $6924 \mathrm{~h}$ led to further degradation of fibrinogen (Figure S4A, lane 6 from left). Figure S4A does not 
reveal the sequence in which MMP1 degrades the three chains of fibrinogen. Figure S4B shows the time-dependent degradation of fibrinogen. MMP1 degrades the $\alpha$-chain first and the $\gamma$-chain last. A comparison with prior research shows that plasmin, the well-known fibrinolytic agent, also degrades the $\alpha$-chain first and the $\gamma$-chain last ${ }^{5}$. Next, we tested the effects of MMP1 on fibrin morphology using SEM (Figure S4C), where the destruction of fibrin structures is visible for active MMP1. We created similar thin layers of fibrin morphologies for smFRET experiments. Overall, the ensemble experiments in Figure S4 show that MMP1 has fibrinolytic activity on fibrinogen and fibrin, and there is a specific sequence of degradation of the three chains. Both thrombin and trypsin cleave the same Arg-Gly bonds in fibrinogen ${ }^{6}$. However, thrombin is highly specific, and only four bonds linking the fibrinopeptides to the rest of the fibrinogen molecule are rapidly hydrolyzed by thrombin, whereas trypsin cleaves all peptide bonds in fibrinogen that 81 follow arginine or lysine ${ }^{6}$.

82 In comparison, degradation of fibrinogen and crosslinked fibrin by MMPs do not have specific cleavage sites across the MMP-family. For example, MMP3, MMP7, and MMP14 cleave the $\alpha$-chain at Asp97-Phe98 and Asn102-Asn103; the $\beta$-chain at Asp123-

88 Leu124, Asn137-Val138, and Glu141-Tyr142; and the $\gamma$-chain at Thr83-Leu84 ${ }^{2}$. Cleavage sites on both fibrinogen and crosslinked fibrin for these three MMPs are close to those for 92 plasmin. Additionally, MMP3 solubilizes 93 crosslinked fibrin by cleaving the Gly404Ala405 bond in the $\gamma$-chain ${ }^{7}$, resulting in a Dlike monomer fragment at $\sim 94 \mathrm{kDa}$ similar to fibrinogen degradation by plasmin. In contrast, MMP7 and MMP14 solubilize crosslinked fibrin and produces D-like dimer fragments at $\sim 186 \quad \mathrm{kDa}$ similar to crosslinked fibrin degradation by plasmin ${ }^{2}$. However, MMP1,

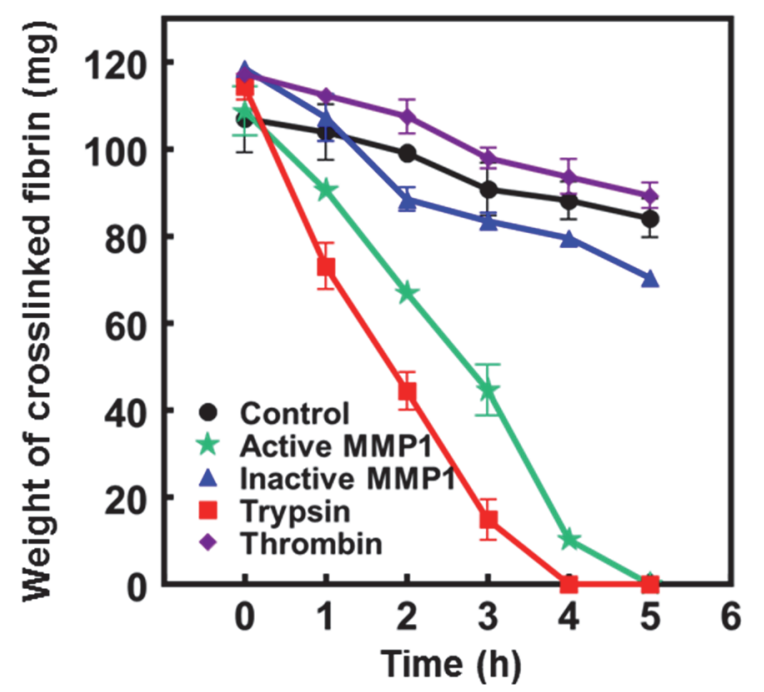

Figure S5. Weight-based fibrin degradation assay. Time-dependent weights of crosslinked fibrin after treatment with active MMP1, active site mutant of MMP1, trypsin, thrombin, and PBS buffer as the control.

102

103

104 MMP2, MMP9, and MMP15 do not show similar activity ${ }^{2}$. Fibrinogen degradation by catalytic domains of MMP8, MMP12, MMP13, and MMP14 show differences in the cleavage sites ${ }^{8}$. Overall, MMPs show differences in fibrinolytic activity, which is intriguing because the catalytic domain has a largely conserved sequence among the MMP-family members 9 . Interestingly, prior research reported insignificant fibrinolytic activity of $\mathrm{MMP}^{7}$, in contrast to our findings.

Weight-based degradation assay for water-insoluble fibrin. We used a weight-based degradation assay to quantify the activity of MMP1 because fibrin is water-insoluble and as such, solution-based biochemical assays are appropriate. We prepared fibrin with crosslinks by mixing $1105 \mu \mathrm{g}$ of human factor XIII, 20 units of thrombin (Cayman chemical, Cat\# 13188), and $40 \mu \mathrm{L}$ of $11110 \mathrm{mM}$ PBS (pH 7.4) in a $0.5 \mathrm{~mL}$ PCR tube at $22^{\circ} \mathrm{C}$. We incubated the mixture for $10 \mathrm{~min}$ at $37^{\circ}$ $112 \mathrm{C}$ without shaking. We added $100 \mu \mathrm{g}$ of fibrinogen (Cayman chemical, Cat\# 16088) and $20 \mu \mathrm{L}$ of $1135 \mathrm{mM} \mathrm{CaCl}_{2}$ to the mixture and incubated for an additional $15 \mathrm{~min}$ at $37^{\circ} \mathrm{C}$ without shaking. After 114 incubation, the solution becomes turbid, indicating the formation of crosslinked fibrin. We 
115 prepared five reactions for crosslinked fibrin and added 1) $100 \mu \mathrm{L}$ of $10 \mathrm{mM}$ PBS (pH 7.4), 2) 100 $116 \mu \mathrm{L}$ of $1 \mathrm{mg} / \mathrm{mL}$ active MMP1, 3) $100 \mu \mathrm{L}$ of $1 \mathrm{mg} / \mathrm{mL}$ active MMP1,4) $100 \mu \mathrm{L}$ of $1 \mathrm{mg} / \mathrm{mL}$ trypsin, 117 and 5) 100 units of thrombin. We made the final volume of each reaction to be $200 \mu \mathrm{L}$ by diluting with $10 \mathrm{mM}$ PBS (pH 7.4). At $0 \mathrm{hr}$, we centrifuged the reactions at $10000 \mathrm{rpm}$ for $10 \mathrm{~min}$ using a tabletop centrifuge, discarded the supernatant, and weighed the tubes. We subtracted the weights of the PCR tubes for each specific reaction before preparing the reactions. For five conditions, we measured weight of $\sim 120 \mathrm{mg}$. After weighing, we again added 1) $100 \mu \mathrm{L}$ of $10 \mathrm{mM}$ PBS (pH 7.4), 2) $100 \mu \mathrm{L}$ of $1 \mathrm{mg} / \mathrm{mL}$ active MMP1, 3) $100 \mu \mathrm{L}$ of $1 \mathrm{mg} / \mathrm{mL}$ active MMP1, 4) $100 \mu \mathrm{L}$ of $1 \mathrm{mg} / \mathrm{mL}$ trypsin, and 5) 100 units of thrombin to a final volume of $200 \mu \mathrm{L}$ by diluting with $10 \mathrm{mM}$ PBS ( $\mathrm{pH}$ 7.4). We incubated the reactions at $37^{\circ} \mathrm{C}$ without shaking. At $1 \mathrm{~h}$, we centrifuged the tubes again, weighed, and subtracted the weight of empty tubes. We diluted again as above and incubated for another hour. We repeated the process at $0 \mathrm{~h}, 1 \mathrm{~h}, 2 \mathrm{~h}, 3 \mathrm{~h}, 4 \mathrm{~h}$, and $5 \mathrm{~h}$. We performed three biological repeats to calculate the mean and standard deviations. Figure S5 shows that active MMP1 and trypsin degrade crosslinked fibrin at a similar rate of $\sim 30 \mathrm{mg} / \mathrm{h}$. Active site mutant of MMP1, thrombin, and control show similar changes in weights at a rate of $\sim 7 \mathrm{mg} / \mathrm{h}$. After 130 subtracting the control, the rate of crosslinked fibrin degradation by MMP1 is $\sim 0.23 \mathrm{mg} / \mathrm{h} / \mu \mathrm{g}$. Note that the weight includes water and, as such, has a higher value.

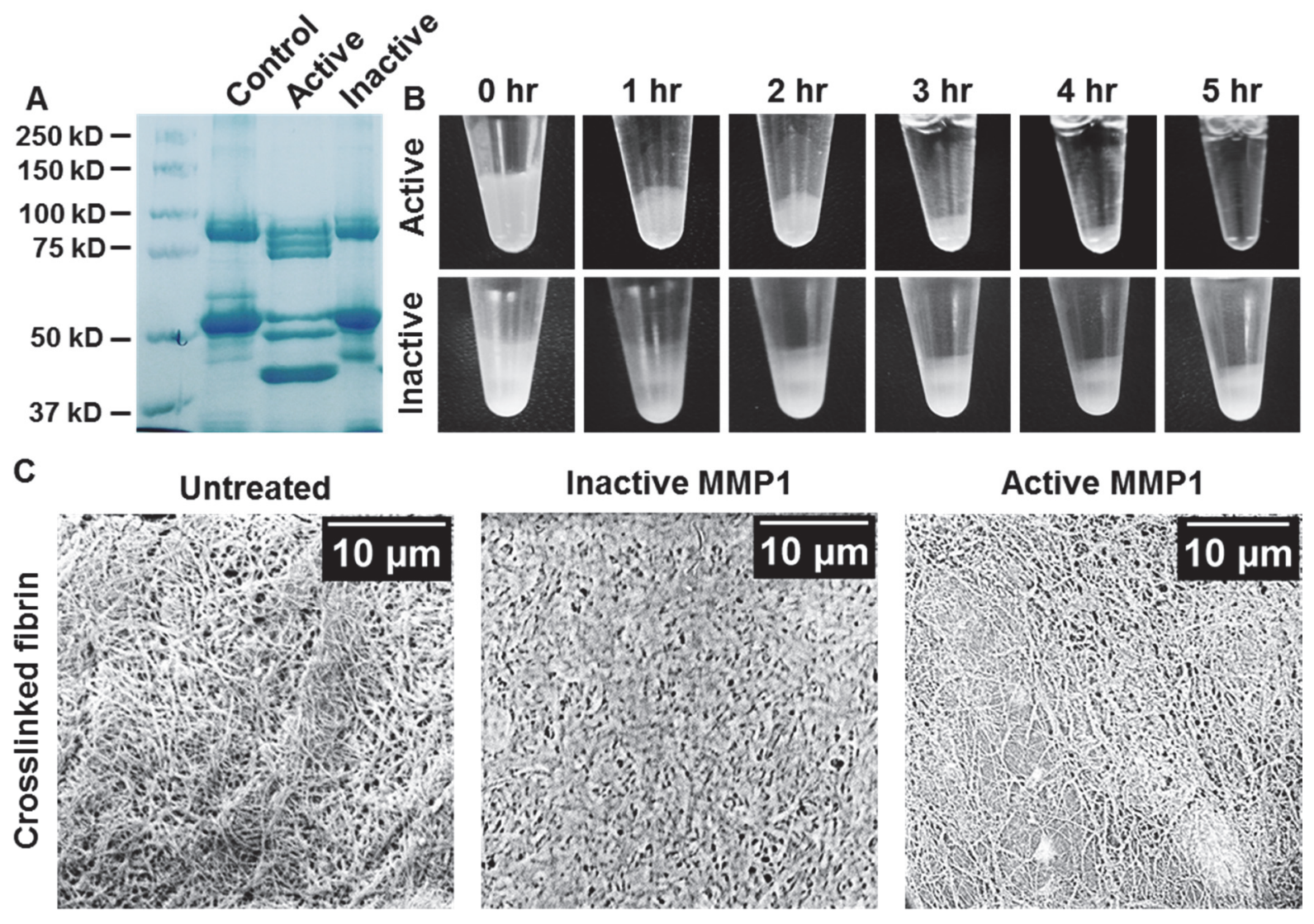

Figure S6. Fibrinolytic activity of MMP1 on crosslinked fibrin. (A) SDS PAGE of crosslinked fibrin with active MMP1 and active site mutant of MMP1. The control uses the protein buffer (50 mM Tris, $100 \mathrm{mM} \mathrm{NaCl}, \mathrm{pH} 8.0$ ). (B) $100 \mathrm{mg}$ of wet crosslinked fibrin treated with $0.1 \mathrm{mg} / \mathrm{mL}$ active MMP1 and active site mutant of MMP1 at $37^{\circ}$ C. (C) SEM images of crosslinked fibrin with and without MMP1 treatment. 
MMP1 activity on crosslinked fibrin. Water-soluble fibrinogen becomes water-insoluble crosslinked fibrin in the presence of factor XIII, thrombin, and $\mathrm{CaCl}_{2}{ }^{10}$. Thrombin converts fibrinogen into fibrin monomer by cleaving fibrinopeptide $\mathrm{A}$ and fibrinopeptide $\mathrm{B}^{11,12}$. Fibrin monomers self-assemble into protofibrils. The polymerization sites noncovalently attach to the D regions of two other fibrin(ogen) molecules. Fibrin monomers in each strand assemble end-to-end, whereas monomers across the strands arrange in a half-staggered overlap. The $\gamma$-chains of adjacent $\mathrm{D}$ regions in each strand is covalently attached by isopeptide bonds, which are formed due to the activity of factor XIIIa and appear as a $\sim 94 \mathrm{kDa}$ band in SDS PAGE under reducing conditions (Figure S6A). Since factor XIIIa does not crosslink the $\beta$-chains ${ }^{13,14}$, the band at $\sim 52 \mathrm{kDa}$ due to the $\beta$-chains remains unchanged in SDS PAGE of fibrinogen (Figure S6A) and crosslinked fibrin (Figure S6A). As shown in Figure S6B, active MMP1 degrades and dissolves water-insoluble crosslinked fibrin, but active site mutant of MMP1 does not dissolve crosslinked fibrin. Figure S6C shows the surface morphology of crosslinked fibrin imaged using SEM. Treatment with active MMP1 resulted in a more porous structure of crosslinked fibrin.

\section{References} Arnold, B. C. Pareto distribution. Wiley StatsRef: Statistics Reference Online, 1-10 (2014). Bini, A., Wu, D., Schnuer, J. \& Kudryk, B. J. Characterization of stromelysin 1 (MMP-3), matrilysin (MMP-7), and membrane type 1 matrix metalloproteinase (MT1-MMP) derived fibrin (ogen) fragments D-dimer and D-like monomer: NH2-terminal sequences of latestage digest fragments. Biochemistry 38, 13928-13936 (1999).

3 Astrup, T. \& Sterndorff, I. Fibrinolytic activity of tissue extracts and of trypsin. Nature 170, 981-981 (1952).

4 Sarkar, S. K., Marmer, B., Goldberg, G. \& Neuman, K. C. Single-molecule tracking of collagenase on native type I collagen fibrils reveals degradation mechanism. Current Biology 22, 1047-1056 (2012).

5 Pizzo, S. V., Schwartz, M. L., Hill, R. L. \& McKee, P. A. The effect of plasmin on the subunit structure of human fibrinogen. Journal of biological chemistry 247, 636-645 (1972).

6 Andreatta, R. H., Liem, R. K. \& Scheraga, H. A. Mechanism of action of thrombin on fibrinogen, I. Synthesis of fibrinogen-like peptides, and their proteolysis by thrombin and trypsin. Proceedings of the National Academy of Sciences 68, 253-256 (1971). Bini, A., Itoh, Y., Kudryk, B. J. \& Nagase, H. Degradation of Cross-Linked Fibrin by Matrix Metalloproteinase 3 (Stromelysin 1): Hydrolysis of the $\gamma$ Gly 404- Ala 405 Peptide Bond. Biochemistry 35, 13056-13063 (1996).

8 Hiller, O., Lichte, A., Oberpichler, A., Kocourek, A. \& Tschesche, H. Matrix metalloproteinases collagenase-2, macrophage elastase, collagenase-3, and membrane type 1-matrix metalloproteinase impair clotting by degradation of fibrinogen and factor XII. Journal of Biological Chemistry 275, 33008-33013 (2000). metalloproteinases. Proceedings of the National Academy of Sciences 111, E4148-E4155 (2014).

10 Weisel, J. W. \& Litvinov, R. I. in Fibrous proteins: structures and mechanisms 405-456 (Springer, 2017).

11 Medved, L. \& Weisel, J. Recommendations for nomenclature on fibrinogen and fibrin. Journal of Thrombosis and Haemostasis 7, 355-359 (2009). 
17812 Weisel, J. W. \& Litvinov, R. I. Mechanisms of fibrin polymerization and clinical 179 implications. Blood, The Journal of the American Society of Hematology 121, 1712-1719 180 (2013).

18113 McKee, P. A., Mattock, P. \& Hill, R. L. Subunit structure of human fibrinogen, soluble

182

183 fibrin, and cross-linked insoluble fibrin. Proceedings of the National Academy of Sciences 66, 738-744 (1970).

14 Sobel, J. H. \& Gawinowicz, M. A. Identification of the $\alpha$ chain lysine donor sites involved in factor XIIIa fibrin cross-linking. Journal of Biological Chemistry 271, 19288-19297 